\title{
Endogeneity in Nonlinear Regressions with Integrated Time Series
}

\author{
Yoosoon Chang ${ }^{1}$ \\ and \\ Joon Y. Park \\ Department of Economics \\ Indiana University
}

\begin{abstract}
This article considers the nonlinear regression with integrated regressors that are contemporaneously correlated with the regression error. We, in particular, establish the consistency and derive the limit distribution of the nonlinear least squares estimator under such endogeneity. For the regressions with various types of regression functions, it is shown that the estimator is consistent and has the same rate of convergence as for the case of the regressions with no endogeneity. Whether or not the limit distribution is affected by the presence of endogeneity, however, depends upon the functional type of the parameter derivative of regression function. If it is asymptotically homogeneous, the limit distribution of the nonlinear least squares estimator has an additional bias term reflecting the presence of endogeneity. On the other hand, the endogeneity does not have any effect on the nonlinear least squares limit theory, if the parameter derivative of regression function is integrable. Regardless of the presence of endogeneity, the least squares estimator has the same limit distribution in this case. To illustrate our theory, we consider the nonlinear regressions with logistic and power regression functions with integrated regressors that have contemporaneous correlations with the regression error.
\end{abstract}

This version: February 15, 2009

JEL Classification: C13, C22.

Key words and phrases: nonlinear regression, integrated time series, endogeneity, consistency, limit distributions.

\footnotetext{
${ }^{1}$ We are grateful to the Editor and two anonymous referees for their useful comments. The corresponding author: Department of Economics, Indiana University, Wylie Hall Rm 105, 100 S. Woodlawn, Bloomington, IN 47405-7104, Tel: 812-855-8035, Email: yoosoon@indiana.edu. An earlier version of this article was presented at the 2004 ASSA meeting, San Diego. Chang and Park gratefully acknowledge the financial support from the NSF under Grant No. SES-0453069/0730152 and SES-0518619, respectively.
} 


\section{Introduction}

The asymptotic theory for nonlinear regressions with integrated regressors has been developed by Park and Phillips (1999, 2001), and extended later by several authors including Park and Phillips (2000), Chang and Park (2003) and Chang et al. (2005), among others. The asymptotic properties of the nonlinear least squares (NLS) estimators are now well known. In particular, the NLS estimators are consistent under very mild conditions. The convergence rates and limit distributions are, however, dependent upon the regression function, especially upon what function class its parameter derivative belongs to. If the parameter derivative of regression function is integrable, the convergence rate is $\sqrt[4]{n}$ and the limit distribution is mixed normal. If, on the other hand, it is asymptotically homogeneous with asymptotic order $\dot{\kappa}$, then the exact convergence rate is given by $\sqrt{n} \dot{\kappa}(\sqrt{n})$ and the limit distribution is generally not mixed normal. The asymptotic results for the regressions with mixtures of integrable and asymptotically homogeneous functions are also available in Chang et al. (2001).

Virtually all the previous work, however, does not allow for contemporaneous correlation between the regressors and regression errors. ${ }^{2}$ The effect of the presence of endogeneity on the nonlinear regression with integrated regressor is therefore largely unknown except for some simple cases. For the linear model with integrated regressors, however, it is well known that the endogeneity only has a second-order effect on its asymptoics. The convergence rate is $n$, regardless of the presence or absence of the contemporaneous correlation between the regressors and regression error. The endogeneity affects the limit distribution, but not in any critical manner. The limit distribution of the ordinary least squares (OLS) estimator is not mixed normal, unless the regressors are strictly exogenous, having zero longrun covariance with the regression error. It is therefore interesting to see whether this extends to the nonlinear regressions with integrated regressors.

In this article, we consider the nonlinear regression with integrated regressors that are contemporaneously correlated with the regression error. In particular, we establish the consistency and obtain the limit distribution of the NLS estimator. We consider both cases where the parameter derivative of regression function is integrable and asymptotically homogeneous. Our results show that the NLS estimator is generally consistent in both cases, even in the presence of endogeneity. Indeed, the NLS estimator has exactly the same rate of convergence as for the regression with no endogeneity. However, whether or not the limit distribution of the NLS estimator is affected by the presence of endogeneity depends upon the types of the parameter derivative of regression function. If it is asymptotically homogeneous, the limit distribution of the NLS estimator has an additional bias term reflecting the endogeneity. If it is integrable, however, the limit distribution of the NLS estimator remains the same, having no additional bias term. The presence of endogeneity does not have any effect even on the limit distribution of the NLS estimator, if the parameter derivative of

\footnotetext{
${ }^{2}$ An exception is de Jong (2002), which independently develops the asymptotics under endogeneity for the regression with regression function that is more explosive than linear function. His model, in particular, does not include the regression with bounded regression function which we believe to be the most interesting case.
} 
regression function is integrable. ${ }^{3}$

There are some important implications of the results we obtained in the article. First, the presence of endogeneity in general does not affect the convergence rate for the NLS estimator. This implies in particular that the nonlinear regression with integrated regressors is not identified by the usual orthogonality condition between the regressors and regression errors. Rather, it is identified by the nonlinear cointegrating relationship, i.e., by the residuals being stationary. Second, it has long been believed that endogeneity does not cause inconsistency in the linear cointegrating regression, mainly because the OLS estimator is super-consistent and converges at a faster rate. Our results in the article clearly show that it is not the faster convergence rate, but the magnitude of the signal provided by the stochastic trend in the integrated regressor, which makes the estimator robust to the presence of endogeneity. Indeed, the signal robustifies the NLS estimator against the presence of endogeneity even more effectively in the regression with regression function having integrable parameter derivative, which has a reduced convergence rate $\sqrt[4]{n}$.

The rest of the article is organized as follows. In Section 2, we introduce the model and assumptions. The function classes and preliminary asymptotics are presented in Section 3. These are the results that we need to develop our main asymptotics. The main asymptotics for the NLS estimator under endogeneity are provided in Section 4. Some specific examples are given in Section 5, which show how our results may be used to obtain the explicit asymptotics for the NLS estimators. In Section 6, we present some simulation results to show the performance of the NLS estimators in finite samples. Our simulation results are largely consistent with the asymptotic results. Section 7 concludes the article.

\section{The Model and Assumptions}

We consider the model given by

$$
y_{t}=f\left(x_{t}, \theta_{0}\right)+\varepsilon_{t}
$$

where $\left(x_{t}\right)$ is a univariate integrated regressor and given by

$$
x_{t}=x_{t-1}+v_{t}
$$

and $\left(\varepsilon_{t}\right)$ is the regression error that we further specify as

$$
\varepsilon_{t}=\sqrt{1-\rho^{2}} u_{t}+\rho v_{t}
$$

for some constant $\rho$ such that $|\rho| \leq 1$.

We assume that the function $f: \mathbb{R} \times \mathbb{R}^{m} \rightarrow \mathbb{R}$ is known, and $\theta_{0}$ is an $m$-dimensional true parameter vector that lies inside the parameter set $\Theta$. Moreover, the following statement is assumed throughout the article, unless stated otherwise, that

\footnotetext{
${ }^{3}$ Strictly speaking, this is only true in our simple i.i.d. setup. In general, it is expected to have a scale effect, as we may well infer from the recent related study by Jaganathan (2008) for dependent processes.
} 
Assumption 2.1 Let $\left(u_{t}\right)$ be independent of $\left(v_{t}\right)$, and independent and identically distributed with mean zero and finite variance $\sigma_{u}^{2}$.

Assumption 2.2 Let $\left(v_{t}\right)$ be independent and identically distributed with mean zero and variance $\sigma_{v}^{2}$, and have finite $\nu$-th moment.

Some of our subsequent results require additional conditions on the distribution of $\left(v_{t}\right)$, which is given by

Assumption 2.3 We assume that the characteristic function $\varphi$ of $\left(v_{t}\right)$ satisfies either (a) $|\varphi(s)|=1$ if and only if $s$ is a multiple of $2 \pi$ or (b) $\int_{-\infty}^{\infty}|\varphi(s)|^{2} d s<\infty$, depending upon whether the distribution of $\left(v_{t}\right)$ is of discrete type or of continuous type.

In the subsequent development of our theory, we denote by $\mu$ the Lebesgue or counting measure, depending upon whether the distribution of $\left(v_{t}\right)$ is of continuous or of discrete type, and the probability density of $\left(v_{t}\right)$ with respect to $\mu$ is signified by $p$. For the expositional simplicity, we assume $\sigma_{u}^{2}=\sigma_{v}^{2}=1$ for the rest of the article. Their variances only have trivial scaling effect, which is unimportant for analyzing the effect of the presence of endogeneity. Under our construction, the variance of $\left(\varepsilon_{t}\right)$ also becomes unity regardless of the value of $\rho$.

The nonlinear regression model in (1) is considered earlier by Park and Phillips (2001). They, however, only consider the models with exogenous errors. This amounts to assuming $\rho=0$ in our specification (3). The lack of contemporaneous correlation between the regressor and the regression error is crucial for the derivation of their results. On the contrary, the regressor and the error are contemporaneously correlated in our model, except for the case of $\rho=0$. In our model, $\rho$ measures the contemporaneous correlation coefficient between the regressor and the regression error. The value of $\rho$ signifies the fraction of the endogenous component of the regression error, and hence, we might say that the degree of endogeneity increases as $|\rho| \rightarrow 1$. The endogeneity becomes maximal when $|\rho|=1$.

Our assumptions on the innovation $\left(v_{t}\right)$ of the regressor are rather stringent, and in particular, stronger than those used in Park and Phillips (2001). Such stringent assumptions are introduced here to highlight and fully analyze the effects of the presence of endogeneity. Our assumption of independent and identical distribution for the innovation $\left(v_{t}\right)$ of the regressor is indeed unnecessarily strong for many of the subsequent results. ${ }^{4}$ Some of them can be derived under a weaker set of conditions, which allow in particular for the presence of serial correlation. Others require some modifications, but they are simple and straightforward. At least qualitatively, we may expect in all cases similar results to hold for more general $\left(v_{t}\right)$. These will be pointed out along the way, as we develop our theory. We may allow $\left(u_{t}\right)$ to be a more general martingale difference sequence with respect to a filtration $\left(\mathcal{F}_{t}\right)$, say, as long as $\left(x_{t}\right)$ is adapted to $\left(\mathcal{F}_{t-1}\right)$.

Although we assume that $\left(v_{t}\right)$ is given by a sequence of independent and identically distributed random variables, the underlying distribution is allowed to be of discrete type,

\footnotetext{
${ }^{4}$ This is clear from Jaganathan (2008) and de Jong (2002), which show that some of our results can be extended to models driven by a dependent process $\left(v_{t}\right)$.
} 
as well as of continuous type. Our assumptions on the underlying distribution are rather mild and hold for a wide class of distributions. The asymptotic theory developed in Park and Phillips $(1999,2001)$ and others on the asymptotics for the integrable transformations of integrated time series all require the underlying distribution to be of continuous type. This is because all the previous works rely on Akonom (1993), while our theory is built upon Borodin (1986). The former gives some basic asymptotic theories for the integrated processes driven by the linear processes having innovations with continuous distributions, but in contrast the latter provides more comprehensive asymptotics for the simple random walks generated by independent and identically distributed innovations with both discrete and continuous distributions.

Here we consider a simple regression, which has only a single regressor. This is solely for expositional purpose. Although simple, the model we introduced in (1) - (3) has all essential features that we need to analyze the effect of the presence of endogeneity in nonlinear regressions with integrated time series. Therefore, it serves our purpose very well. Our subsequent results are applicable not only for the model considered here, but also for a wide class of more general nonlinear regression models with various additional regressors, stationary as well as integrated, deterministic as well as stochastic. The required extension is indeed rather straightforward and can easily be done following the earlier work by Chang et al. (2001). The details of the results for more general models will be given as we develop the theory for our simple model.

In the article, we consider the estimation of (1) by nonlinear least squares (NLS). If we let

$$
Q_{n}(\theta)=\sum_{t=1}^{n}\left(y_{t}-f\left(x_{t}, \theta\right)\right)^{2}
$$

then the NLS estimator $\hat{\theta}_{n}$ of $\theta$ is defined as the minimizer of $Q_{n}(\theta)$ over $\theta \in \Theta$, i.e.,

$$
\hat{\theta}_{n}=\underset{\theta \in \Theta}{\operatorname{argmin}} Q_{n}(\theta)
$$

An error variance estimate is given by

$$
\hat{\sigma}_{n}^{2}=\frac{1}{n} \sum_{t=1}^{n} \hat{\varepsilon}_{t}^{2}
$$

where $\hat{\varepsilon}_{t}=y_{t}-f\left(x_{t}, \hat{\theta}_{n}\right)$.

Under Assumptions 2.1 and 2.2 with $\nu=2$, an invariance principle holds jointly for $\left(u_{t}\right)$ and $\left(v_{t}\right)$. In particular, if we let $U_{n}(r)=n^{-1 / 2} \sum_{t=1}^{[n r]} u_{t}$ and $V_{n}(r)=n^{-1 / 2} \sum_{t=1}^{[n r]} v_{t}$, then we have

$$
\left(U_{n}, V_{n}\right) \rightarrow_{d}(U, V)
$$

where $U$ and $V$ are independent standard Brownian motions. These are the Brownian motions that we use to represent the asymptotics for $\hat{\theta}_{n}$. Using the so-called Skorohod representation theorem, we may redefine $\left(U_{n}, V_{n}\right)$ up to the distributional equivalence so that $\left(U_{n}, V_{n}\right)$ and $(U, V)$ are defined on a common probability space, and $\left(U_{n}, V_{n}\right) \rightarrow_{a . s .}(U, V)$. This is well known. The stochastic process $\left(U_{n}, V_{n}\right)$ takes values in $D[0,1]^{2}$, where $D[0,1]$ 
is the space of cadlag functions defined on the unit interval $[0,1]$. For our purpose, it is convenient to endow $D[0,1]$ with the uniform metric.

Our limit theory also involves the local time $L$ of the Brownian motion $V$, which is given by

$$
L(t, x)=\lim _{\epsilon \rightarrow 0} \frac{1}{2 \epsilon} \int_{0}^{t} 1\{|V(s)-x|<\epsilon\} d s
$$

Roughly, $L(t, x)$ measures the rate of time spent by $V$ in a neighborhood of $x$, up to time $t$. In our subsequent theory, in particular, frequently appears $L(1,0)$, i.e., the rate of time spent by $V$ in an immediate vicinity of the origin in the unit time interval. The reader is referred for the details of the Brownian local time $L$ to Park and Phillips (1999) and the references cited there.

\section{Function Classes and Preliminary Asymptotics}

In this section, we introduce the function classes and their asymptotics that are needed for the development of our theory on nonlinear regressions with integrated time series.

\subsection{Regular and Strongly Regular Functions}

First we consider the asymptotics for normalized integrated time series, and introduce the required regularity conditions. Obviously, the presence of endogeneity only affects the covariance asymptotics, which we will mostly look at here. The same results as in Park and Phillips (2001, PP henceforth) apply for the mean asymptotics. ${ }^{5}$

Definition 3.1 A transformation $T$ on $\mathbb{R}$ is said to be regular if

(a) it is locally bounded and Riemann-integrable, ${ }^{6}$ and

(b) it is (i) bounded by $c|x|^{a}$ for all $x \in \mathbb{R} \backslash\{0\}$ in a neighborhood of the origin for some constants $a>-1$ and $c>0$, and (ii) continuously differentiable for all $x \in \mathbb{R} \backslash\{0\}$ with derivative bounded by $c|x|^{b}$ for some constants $b$ and $c>0$ in a neighborhood of the origin.

If, in addition to (a) and (b),

(c) it either has vanishing derivative on $\mathbb{R} \backslash\{0\}$ or is continuous at the origin, then it is said to be strongly regular.

Lemma 3.2 Suppose that Assumptions 2.2 with $\nu=4$ and 2.3 hold, and let $T$ be a transformation on $\mathbb{R}$.

(a) If $T$ is regular, then

$$
\frac{1}{\sqrt{n}} \sum_{t=1}^{n} T\left(\frac{x_{t}}{\sqrt{n}}\right) v_{t}=O_{p}(1)
$$

\footnotetext{
${ }^{5}$ As in PP, we define mean asymptotics as the asymptotics for sample means of nonlinear transformations of integrated time series, and covariance asymptotics as the asymptotics for sample product moments of nonlinear transformation of integrated time series and stationary innovations.

${ }^{6}$ More precisely, we require that the Riemann-integral $\int_{K} T(x) d x$ of $T$ exists and is finite over any compact interval $K$ including the origin.
} 
for all large $n$.

(b) Let $T$ be strongly regular. If $T$ has vanishing derivative on $\mathbb{R} \backslash\{0\}$ and has values $a$ and $b$ respectively on the positive and negative parts of $\mathbb{R}$, then

$$
\frac{1}{\sqrt{n}} \sum_{t=1}^{n} T\left(\frac{x_{t}}{\sqrt{n}}\right) v_{t} \rightarrow_{d} \int_{0}^{1} T(V(r)) d V(r)+(b-a) L(1,0)
$$

as $n \rightarrow \infty$.

(c) Let $T$ be strongly regular. If $T$ is continuous at the origin and has nonvanishing derivative $\nabla T$ on $\mathbb{R} \backslash\{0\}$, then

$$
\frac{1}{\sqrt{n}} \sum_{t=1}^{n} T\left(\frac{x_{t}}{\sqrt{n}}\right) v_{t} \rightarrow_{d} \int_{0}^{1} T(V(r)) d V(r)+\int_{0}^{1} \nabla T(V(r)) d r
$$

as $n \rightarrow \infty$.

The motivations for our definitions of regular and strongly regular functions are now clear. For all regular functions, the cross product sum of the properly normalized and transformed integrated process with its contemporaneous innovation is shown to be of order $O_{p}\left(n^{1 / 2}\right)$. The regularity conditions are therefore sufficient to ensure that the required normalization factor is $\sqrt{n}$ for the covariance asymptotics under endogeneity. With the strong regularity conditions, we may obtain the explicit covariance asymptotics in the presence of endogeneity. The regularity conditions required for the regular and strongly regular functions may seem stringent. However, they are satisfied by virtually all functions that are used in practical nonlinear analyses. They hold, for instance, for all the examples considered in PP.

It can be easily deduced, e.g. as in PP, that

$$
\frac{1}{\sqrt{n}} \sum_{t=1}^{n} T\left(\frac{x_{t}}{\sqrt{n}}\right) u_{t} \rightarrow_{d} \int_{0}^{1} T(V(r)) d U(r)
$$

as $n \rightarrow \infty,,^{7}$ which may be regarded as the covariance asymptotics without endogeneity. Comparing (7) with our results in Lemma 3.2, we may readily see that our regularity conditions are sufficient to guarantee that the same $\sqrt{n}$-rate is applicable for the covariance asymptotics even in the presence of endogeneity. If some additional conditions are met and the strong regularity holds, the covariance asymptotics comparable to (7) are derived. The presence of endogeneity affects the covariance asymptotics, producing an additional term reflecting its presence. For instance, respectively for $T(x)=1\{x \geq 0\}$ and $T(x)=x^{2}$, it

\footnotetext{
${ }^{7} \mathrm{PP}$ establishes the result under weaker martingale difference assumption on $\left(u_{t}\right)$. It also uses a slightly different set of regularity conditions. The regularity concept in PP is equivalent to the local boundedness and local Riemann-integrability here. For their equivalence, see the proof of Theorem 1.1, pp. 81-82, in Borodin and Ibragimov (1995).
} 
follows from Lemma 3.2 that

$$
\begin{array}{r}
n^{-1 / 2} \sum_{t=1}^{n} 1\left\{x_{t} \geq 0\right\} v_{t} \rightarrow \int_{0}^{1} 1\{V(r) \geq 0\} d V(r)+L(1,0) \\
n^{-3 / 2} \sum_{t=1}^{n} x_{t}^{2} v_{t} \rightarrow_{d} \int_{0}^{1} V^{2}(r) d V(r)+2 \int_{0}^{1} V(r) d r
\end{array}
$$

as $n \rightarrow \infty$. Note that for the case of $\nabla T=0$ the additional bias term does not vanish unless $a=b$, i.e., the function $T$ is essentially constant.

In the covariance asymptotics for the strongly regular functions, it is interesting to note that the stochastic processes

$$
L(r, 0) \text { and } \quad \int_{0}^{r} \nabla T(V(s)) d s
$$

are additive functionals whose sample paths are of bounded variation a.s., while

$$
\int_{0}^{r} T(V(s)) d V(s)
$$

is a continuous martingale. The limit processes are therefore given by semi-martingales for the covariance asymptotics under endogeneity. This is in contrast to the exogenous case, where the limit process is represented solely by a continuous martingale. The presence of the bounded variation components, of course, implies that their limit distributions are biased. The presence of endogeneity thus introduces bias in the limit distributions of the covariance asymptotics.

So far, we have assumed that the transformation $T$ is real-valued. We may, however, easily extend our definitions and results to the vector-valued function $T$. From now on, we will say that a vector-valued function $T$ is regular (strongly regular) if all of its component real-valued functions are regular (strongly regular) in the sense of Definition 3.1. For such vector-valued functions, the covariance asymptotics obtained in Lemma 3.2 continue to hold. Of course, in this case, the integrals $\int_{0}^{1} T(V(r)) d V(r)$ and $\int_{0}^{1} \nabla T(V(r)) d r$ are understood to be vector-valued, and $a$ and $b$ to be vectors. It should however be emphasized that we continue to assume in this article that there is a single integrated time series $\left(x_{t}\right)$ and $V$ is a scalar.

\subsection{I-Regular and H-Regular Functions}

We now consider the family of functions defined on a parameter set, say, $\Pi$. Let $F$ be a vector-valued function defined on $\mathbb{R} \times \Pi$. We introduce two classes of such families: Iand H-regular functions. As in PP, I-regular function is a family of integrable functions satisfying certain regularity conditions, and H-regular function is a family of asymptotically homogeneous functions with required regularity conditions. The asymptotically homogeneous functions, introduced first by Park and Phillips (1999), are the functions that behave as homogeneous functions asymptotically. The parameter set $\Pi$ may be a singleton set, in which case I- and H-regularities become the characteristics of functions rather than families of functions. 
Definition 3.3 We say that $F$ is I-regular on $\Pi$ if

(a) for all $\pi_{0} \in \Pi$, there exists a neighborhood $N_{0}$ of $\pi_{0}$ such that $\left\|F(x, \pi)-F\left(x, \pi_{0}\right)\right\| \leq$ $\left\|\pi-\pi_{0}\right\| T(x)$ for all $\pi \in N_{0}$, where $T: \mathbb{R} \rightarrow \mathbb{R}$ satisfies $|T(x)|<c\left(1+|x|^{-1-\epsilon}\right)$ for some $c>0$ and $\epsilon>0$ and $\int|T(x)| \mu(d x)<\infty$, and

(b) for all $\pi \in \Pi, F(\cdot, \pi)$ is bounded and $\int|x|^{1 / 2+\epsilon}\|F(x, \pi)\| \mu(d x)<\infty$ for some $\epsilon>0$.

Definition 3.4 Let

$$
F(\lambda x, \pi)=\kappa(\lambda, \pi) H(x, \pi)+R(x, \lambda, \pi)
$$

where $\kappa$ is nonsingular. We say that $F$ is H-regular on $\Pi$ if

(a) $H(\cdot, \pi)$ is strongly regular for each $\pi \in \Pi$, and for all $x \in \mathbb{R}, F(x, \cdot)$ is equicontinuous in a neighborhood of $x$, and

(b) $\|R(x, \lambda, \pi)\| \leq \varpi(\lambda, \pi) Q(x)$, where $\varpi(\lambda, \pi)$ is such that $\left(\kappa^{-1} \varpi\right)(\lambda, \pi) \rightarrow 0$ uniformly in $\pi \in \Pi$ as $\lambda \rightarrow \infty$ and $Q$ is regular.

We call $\kappa$ the asymptotic order and $H$ the limit homogeneous function of $F$. If $\kappa$ does not depend upon $\pi$, then $F$ is said to be $H_{0}$-regular.

Definitions 3.3 and 3.4 are comparable to Definitions 3.3 and 3.5, respectively, in PP. The required conditions are, however, somewhat different. Here we modified the conditions in PP so that we may accommodate the integrated processes driven by innovations having discrete distributions and effectively deal with endogeneity. Our conditions for the I-regularity in Definition 3.3 are similar to those used in Definition 3.3 of PP. The differences are mainly due to the necessary modifications that we need in order to use the results of Borodin (1986), in place of Akonom (1993). The conditions for the H-regularity in Definition 3.4 are more distinctive from those in Definition 3.5 of PP. In this article, we impose the strong regularity on the limit homogeneous function to derive the limit distributions under endogeneity. Moreover, here we do not allow for the presence of integral functions in the residual term. This is just to simplify the exposition of our theory. Any integral component included in the asymptotically homogeneous function becomes negligible in the limit, and hence, it can be ignored in our asymptotic analyses.

Though our regularity conditions are different from those used in PP, we may easily deduce the mean and covariance asymptotics in PP for I- and H-regular functions. If $F$ is I-regular on a compact set $\Pi$, then

$$
\frac{1}{\sqrt{n}} \sum_{t=1}^{n} F\left(x_{t}, \pi\right) \rightarrow p L(1,0) \int_{-\infty}^{\infty} F(x, \pi) \mu(d x)
$$

uniformly in $\pi \in \Pi$. Moreover, if $F(\cdot, \pi)$ is I-regular,

$$
\frac{1}{\sqrt[4]{n}} \sum_{t=1}^{n} F\left(x_{t}, \pi\right) u_{t} \rightarrow_{d} \mathbb{M N}\left(0, L(1,0) \int_{-\infty}^{\infty} F(x, \pi) F(x, \pi)^{\prime} \mu(d x)\right)
$$

The mean and covariance asymptotics in (8) and (9) require that Assumptions 2.1, 2.2 with $\nu=3$ and 2.3 hold. 
Similarly, if $F$ is H-regular on a compact set $\Pi$, then

$$
\frac{1}{n} \kappa(\sqrt{n}, \pi)^{-1} \sum_{t=1}^{n} F\left(x_{t}, \pi\right) \rightarrow a . s . \int_{0}^{1} H(V(r), \pi) d r
$$

uniformly in $\pi \in \Pi$. Moreover, if $F(\cdot, \pi)$ is H-regular, then we have

$$
\frac{1}{\sqrt{n}} \kappa(\sqrt{n}, \pi)^{-1} \sum_{t=1}^{n} F\left(x_{t}, \pi\right) u_{t} \rightarrow_{d} \int_{0}^{1} H(V(r), \pi) d U(r)
$$

The mean and covariance asymptotics in (10) and (11) hold under Assumptions 2.1 and 2.2 with $\nu=2$.

The mean and covariance asymptotics in (8) - (11) hold under much more general conditions than we use in this article. The mean and covariance asymptotics in (8) and (9) can be deduced for $\left(x_{t}\right)$ driven by general linear processes as long as the distributions of the innovations satisfy a mild set of regularity conditions, and $\left(u_{t}\right)$ is a martingale difference sequence with respect to the filtration $\left(\mathcal{F}_{t}\right)$ such that $\left(x_{t}\right)$ is adapted to $\left(\mathcal{F}_{t-1}\right)$. Also, we may derive the mean and covariance asymptotics in (10) and (11) under the minimal condition to ensure the weak convergence in (6) and the martingale difference assumption on $\left(u_{t}\right)$ given above. The reader is referred to PP for the details.

We now present the covariance asymptotics for I- and H-regular functions under endogeneity.

Lemma 3.5 Let Assumptions 2.1, 2.2 with $\nu=3$ and 2.3 hold. If $F(\cdot, \pi)$ is I-regular,

$$
\frac{1}{\sqrt[4]{n}} \sum_{t=1}^{n} F\left(x_{t}, \pi\right) v_{t} \rightarrow_{d} \mathbb{M N}\left(0, L(1,0) \int_{-\infty}^{\infty} F(x, \pi) F(x, \pi)^{\prime} \mu(d x)\right)
$$

as $n \rightarrow \infty$, independently of (9).

Lemma 3.6 Let Assumptions 2.1 and 2.2 with $\nu=4$ hold. If $F(\cdot, \pi)$ is H-regular, then we have as $n \rightarrow \infty$

$$
\begin{aligned}
\frac{1}{\sqrt{n}} \sum_{t=1}^{n} F\left(x_{t}, \pi\right) v_{t} & \rightarrow \int_{0}^{1} H(V(r), \pi) d V(r)+(b-a) L(1,0) \\
\frac{1}{\sqrt{n}} \kappa(\sqrt{n}, \pi)^{-1} \sum_{t=1}^{n} F\left(x_{t}, \pi\right) v_{t} & \rightarrow \int_{0}^{1} H(V(r), \pi) d V(r)+\int_{0}^{1} \nabla H(V(r), \pi) d r
\end{aligned}
$$

in the notation introduced in Lemma 3.2, depending upon whether the limit homogeneous function $H(\cdot, \pi)$ has vanishing or nonvanishing derivative on $\mathbb{R} \backslash\{0\}$.

The conditions that we impose to obtain the results in Lemmas 3.5 and 3.6 are far from being necessary. In particular, our assumptions on $\left(v_{t}\right)$ are overly restrictive, and we may well expect that similar results hold for models driven by more general dependent process 
$\left(v_{t}\right)$. In fact, Jaganathan (2008) has recently shown that we have asymptotic results similar to Lemma 3.5 for models with general linear process $\left(v_{t}\right)$. The presence of dependency in $\left(v_{t}\right)$ only has a scale effect, without introducing an additional bias term. His results are very general, allowing the limit process for the normalized $\left(v_{t}\right)$ to be a linear fractional stable motion. ${ }^{8}$ Likewise, de Jong (2002) shows that the second result in Lemma 3.6 can be extended to models with more general stationary dependent process $\left(v_{t}\right)$. The dependency affects the asymptotic result also in this case. However, once again, we have the same bias term scaled only by the one-way longrun variance of $\left(v_{t}\right)$.

\section{Nonlinear Least Squares Asymptotics under Endogeneity}

We now consider the asymptotics for the NLS estimator $\hat{\theta}_{n}$ of $\theta$ defined in (4) in the presence of the contemporaneous cross correlation between the regressor and the regression error. To present our asymptotics, we denote by $\dot{f}$ and $\ddot{f}$ the first- and second-order derivatives of the regression function $f$ with respect to the parameter $\theta$. We assume that they are all vectorized and arranged by lexicographic ordering of their indices. For H-regular $f$, we denote by $\dot{\kappa}$ and $\ddot{\kappa}$ the asymptotic orders of $\dot{f}$ and $\ddot{f}$, respectively, and signify by $\dot{h}$ the limit homogeneous function of $\dot{f}$.

For the regressions with integrable regression functions, we have

Theorem 4.1 Let Assumptions 2.1, 2.2 with $\nu=3$, and 2.3 hold. Assume
(a) $f, \dot{f}$ and $\ddot{f}$ are I-regular on $\Theta$,
(b) $\int_{-\infty}^{\infty}\left(f(x, \theta)-f\left(x, \theta_{0}\right)\right)^{2} \mu(d x)>0$ for all $\theta \neq \theta_{0}$, and
(c) $\int_{-\infty}^{\infty} \dot{f}\left(x, \theta_{0}\right) \dot{f}\left(x, \theta_{0}\right)^{\prime} \mu(d x)>0$.

Then we have

$$
\sqrt[4]{n}\left(\hat{\theta}_{n}-\theta_{0}\right) \rightarrow{ }_{d} \mathbb{M N}\left(0,\left[L(1,0) \int_{-\infty}^{\infty} \dot{f}\left(x, \theta_{0}\right) \dot{f}\left(x, \theta_{0}\right)^{\prime} \mu(d x)\right]^{-1}\right)
$$

and $\hat{\sigma}_{n}^{2} \rightarrow p \sigma^{2}$, as $n \rightarrow \infty$.

For the regressions with I-regular regression functions, Theorem 4.1 shows that the NLS estimator $\hat{\theta}_{n}$ is consistent with the convergence rate $\sqrt[4]{n}$, and has a normal mixture limiting distribution, even under the presence of endogeneity. The consistency of $\hat{\sigma}_{n}^{2}$ is also established. The required regularity conditions are mild. The reader is referred to PP for a detailed discussions on them with some concrete examples.

It should be noted that the asymptotics given in Theorem 4.1 are indeed exactly the same as those obtained by PP for the regressions without endogeneity. The limiting distributions of $\hat{\theta}_{n}$ are independent of $\rho$, the parameter which measures the contemporaneous correlation between the regressor and the regression error. For all the values of $\rho, \hat{\theta}_{n}$ converges to its

\footnotetext{
${ }^{8} \mathrm{His}$ results, however, require $\left(v_{t}\right)$ to have continuous distributions. In contrast, we rely on the approach by Borodin (1986), which allows for both discrete and continuous distributions.
} 
true value $\theta_{0}$ at the same rate. Moreover, if properly standardized, $\hat{\theta}_{n}$ converges to a well defined limiting distribution, which does not depend upon the value of $\rho$. Not surprisingly, endogeneity also has no effect on the consistency of $\hat{\sigma}_{n}^{2}$. We may therefore see that the presence of endogeneity does not play any role, at least for the first order asymptotics, on the asymptotic behaviors of the NLS estimators if the regression function is integrable. ${ }^{9}$

For the regressions with homogeneous regression functions, we have

Theorem 4.2 Let Assumptions 2.1, and 2.2 with $\nu=4$ hold. Assume

(a) $f, \dot{f}$ and $\ddot{f}$ are $\mathrm{H}_{0}$-regular on $\Theta$,

(b) $\kappa>0$ at infinity, $\left\|(\dot{\kappa} \otimes \dot{\kappa})^{-1} \kappa \ddot{\kappa}\right\|<\infty$,

(c) $\int_{|x| \leq \delta}\left(h(x, \theta)-h\left(x, \theta_{0}\right)\right)^{2} d x>0$ for all $\theta \neq \theta_{0}$ and $\delta>0$, and

(d) $\int_{|x| \leq \delta} \dot{h}\left(x, \theta_{0}\right) \dot{h}\left(x, \theta_{0}\right)^{\prime} d x>0$ for all $\delta>0$.

Then we have

$$
\sqrt{n}\left(\hat{\theta}_{n}-\theta_{0}\right) \rightarrow_{d}\left(\int_{0}^{1} \dot{h}\left(V, \theta_{0}\right) \dot{h}\left(V, \theta_{0}\right)^{\prime}\right)^{-1}\left(\int_{0}^{1} \dot{h}\left(V, \theta_{0}\right) d W(\rho)+\rho(b-a) L(1,0)\right)
$$

or

$$
\sqrt{n} \dot{\kappa}(\sqrt{n})^{\prime}\left(\hat{\theta}_{n}-\theta_{0}\right) \rightarrow_{d}\left(\int_{0}^{1} \dot{h}\left(V, \theta_{0}\right) \dot{h}\left(V, \theta_{0}\right)^{\prime}\right)^{-1}\left(\int_{0}^{1} \dot{h}\left(V, \theta_{0}\right) d W(\rho)+\rho \int_{0}^{1} \nabla \dot{h}\left(V, \theta_{0}\right)\right)
$$

where

$$
W(\rho)=\sqrt{1-\rho^{2}} U+\rho V
$$

depending upon whether $\dot{h}\left(\cdot, \theta_{0}\right)$ has vanishing or nonvanishing derivative on $\mathbb{R} \backslash\{0\}$, and $\hat{\sigma}_{n}^{2} \rightarrow p \sigma^{2}$, as $n \rightarrow \infty$.

Theorem 4.3 Let Assumptions 2.1, and 2.2 with $\nu=4$ hold. Assume

(a) $\dot{f}$ is H-regular on $\Theta$,

(b) for any $M>0$ given, there exists $\epsilon>0$ and $\delta$-neighborhood $N$ of $\theta_{0}$ such that

$$
\lambda^{-1+\epsilon}\left\|\dot{\kappa}\left(\lambda, \theta_{0}\right)^{-1}\right\| \rightarrow 0, \quad \lambda^{\epsilon}\left\|(\dot{\kappa} \otimes \dot{\kappa})\left(\lambda, \theta_{0}\right)^{-1}\left(\sup _{|x| \leq M} \sup _{\theta \in N}|\ddot{f}(\lambda x, \theta)|\right)\right\| \rightarrow 0
$$

as $\lambda \rightarrow \infty$, and

(c) $\int_{|x| \leq \delta} \dot{h}\left(x, \theta_{0}\right) \dot{h}\left(x, \theta_{0}\right)^{\prime} d x>0$ for all $\delta>0$.

Then

$$
\sqrt{n}\left(\hat{\theta}_{n}-\theta_{0}\right) \quad \text { or } \quad \sqrt{n} \dot{\kappa}\left(\sqrt{n}, \theta_{0}\right)^{\prime}\left(\hat{\theta}_{n}-\theta_{0}\right)
$$

has the same limiting distributions as in Theorem 4.2, depending upon whether $\dot{h}\left(\cdot, \theta_{0}\right)$ has vanishing or nonvanishing derivative on $\mathbb{R} \backslash\{0\}$, and $\hat{\sigma}_{n}^{2} \rightarrow p \sigma^{2}$, as $n \rightarrow \infty$.

\footnotetext{
${ }^{9}$ Of course, the equivalence we establish here is asymptotic. The endogeneity might still have some significant effects in finite samples. This will be investigated through simulations in Section 6 .
} 
Theorems 4.2 and 4.3 provide the asymptotics for the NLS estimators $\hat{\theta}_{n}$ and $\hat{\sigma}_{n}^{2}$ for the regressions with H-regular regression functions. They both yield the same asymptotics. For the regressions with $\mathrm{H}_{0}$-regular regression functions, the conditions in Theorem 4.2 are required for the derived asymptotics to hold. If the regressions have more general $\mathrm{H}$ regular regression functions, the conditions in Theorem 4.3 need to be satisfied. For a detailed discussions on those conditions and examples, see PP. In short, virtually all the nonlinear regression models used in practical applications satisfy the regularity conditions in Theorems 4.2 or 4.3 .

Unlike the regressions with I-regular regression functions, the presence of endogeneity affects the asymptotics here. The NLS estimator $\hat{\theta}_{n}$ is consistent and the convergence rate remains the same, regardless of the value of the endogeneity parameter $\rho$. We also have the consistency of $\hat{\sigma}_{n}^{2}$ despite of the contemporaneous correlation between the regressor and the regression error. The asymptotic distribution of $\hat{\theta}_{n}$, however, is given as a function of $\rho$. An additional term, which reflects the presence of endogeneity, appears in the limit distribution of $\hat{\theta}_{n}$ as a result of endogeneity. The added term, as we discussed earlier in the previous section, contributes more towards the bias in its asymptotic distribution.

It should be pointed out that the consistency of the NLS estimator $\hat{\theta}_{n}$ here does not necessarily require an accelerated convergence rate. As is well known, the least squares estimator is consistent under endogeneity in linear cointegrating regression model. This, however, has been understood as being due to the super-consistency of the least squares estimator, i.e., the convergence rate being $n$, an order of magnitude greater than the usual $\sqrt{n}$-rate. Our results here make it clear that the consistency still holds under endogeneity when this is not the case. For the regressions with integrated time series, the consistency continues to hold under endogeneity for the regressions with I-regular regression functions or H-regular regression functions with vanishing or non-vanishing derivatives, for which the convergence rate is $\sqrt{n}$ or even slower and reduced to $\sqrt[4]{n}$. The robustness of the consistency in the regressions with integrated time series is not due to the accelerated convergence rate, but to the magnitude of the signal provided by the presence of the stochastic trend in the integrated regressor.

Nonlinear regressions with multiple regressors in an additive regression function are considered in Chang et al. (2001). They consider nonlinear functions of stationary regressors and deterministic trends, as well as integrated regressors, and study how individual components interact in the limit. For instance, they show that in the regression

$$
y_{t}=\sum_{i \in \mathrm{I}} a_{i}\left(x_{i t}, \theta_{i}\right)+\sum_{i \in \mathrm{H}} b_{i}\left(x_{i t}, \theta_{i}\right)+u_{t}
$$

the I-regular terms in the regression function, i.e., $\sum_{i \in \mathrm{I}} a_{i}\left(x_{i t}, \theta_{i}\right)$, are asymptotically orthogonal to the H-regular terms, i.e., $\sum_{i \in \mathrm{H}} b_{i}\left(x_{i t}, \theta_{i}\right)$, and the individual components $a_{i}\left(x_{i t}, \theta_{i}\right)$ in the I-regular terms are orthogonal to each other. This implies that the NLS estimators $\hat{\theta}_{i n}$ of $\theta_{i}$, for $i \in \mathrm{I}$, obtained from running the regression (12) are aymptotically equivalent to the NLS estimators $\tilde{\theta}_{i n}$ of $\theta_{i}$ obtained from running $y_{t}$ on $a_{i}\left(x_{i t}, \theta_{i}\right)$ separately for each $i \in \mathrm{I}$. The asymptotic equivalence between $\hat{\theta}_{i n}$ and $\tilde{\theta}_{i n}$ for the I-regular components provides a useful implication. 
For I-regular regression functions, the individual components $\dot{f}_{i}$ 's of $\dot{f}$ are asymptotically orthogonal to each other as shown in Chang et al. (2001). This in particular implies that the $(m \times m)$ matrix $\int_{-\infty}^{\infty} \dot{f}\left(x, \theta_{0}\right) \dot{f}\left(x, \theta_{0}\right)^{\prime} \mu(d x)$ appearing in the definition of the limit mixing variate of the mixed normal distribution given in Theorem 4.1 is diagonal. The limit distribution given in Theorem 4.1 therefore reduces to

$$
\sqrt[4]{n}\left(\hat{\theta}_{i n}-\theta_{i 0}\right) \rightarrow_{d} \mathbb{M N}\left(0,\left[L(1,0) \int_{-\infty}^{\infty} \dot{f}_{i}\left(x, \theta_{0}\right)^{2} \mu(d x)\right]^{-1}\right)
$$

for each individual component $i=1, \ldots, m$.

\section{Specific Examples}

In this section, we consider some specific nonlinear regression models and obtain the explicit asymptotics as illustrations. We consider two regression functions that seem to be of some special interests in econometric applications. One is the power function given by

$$
f(x, \alpha, \beta)=\alpha x^{\beta} 1\{x>0\}
$$

and the other is the logistic function specified as

$$
f(x, \alpha, \beta)=\alpha e^{\beta x} /\left(1+e^{\beta x}\right)
$$

Following our convention, let $\theta=(\alpha, \beta)^{\prime}$ be the parameter vector, and denote by $\hat{\theta}_{n}=$ $\left(\hat{\alpha}_{n}, \hat{\beta}_{n}\right)^{\prime}$ and $\theta_{0}=\left(\alpha_{0}, \beta_{0}\right)^{\prime}$ the NLS estimator and the true value, respectively.

It is straightforward to show that the power function $f$ given in (14) is H-regular with asymptotic order $\kappa$ and limit homogeneous function $h$ given respectively by

$$
\kappa(\lambda, \alpha, \beta)=\alpha \lambda^{\beta} \quad \text { and } \quad h(x, \alpha, \beta)=x^{\beta} 1\{x>0\}
$$

Moreover, we have

$$
\dot{\kappa}(\lambda, \alpha, \beta)=\left(\begin{array}{ll}
\lambda^{\beta} & 0 \\
\alpha \lambda^{\beta} \log \lambda & \alpha \lambda^{\beta}
\end{array}\right)
$$

and

$$
\dot{h}(x, \alpha, \beta)=\left(\begin{array}{c}
x^{\beta} \\
x^{\beta} \log x
\end{array}\right) 1\{x>0\}, \nabla \dot{h}(x, \alpha, \beta)=\left(\begin{array}{c}
\beta x^{\beta-1} \\
x^{\beta-1}(1+\beta \log x)
\end{array}\right) 1\{x>0\}
$$

If we let $\Theta$ to be a compact subset of $\mathbb{R} \backslash\{0\} \times \mathbb{R}_{+}$, then we may easily check that all the conditions in Theorem 4.3 are satisfied for the power function. We may therefore obtain the limiting distribution of $\hat{\theta}_{n}$ readily from Theorem 4.3.

In particular, if we let $\dot{h}=\left(\dot{h}_{1}, \dot{h}_{2}\right)^{\prime}$ and $\nabla \dot{h}=\left(\nabla \dot{h}_{1}, \nabla \dot{h}_{2}\right)^{\prime}$, and subsequently define

$$
\begin{aligned}
M_{i j} & =\int_{0}^{1} \dot{h}_{i}\left(V, \theta_{0}\right) \dot{h}_{j}\left(V, \theta_{0}\right) \\
N_{i}(\rho) & =\int_{0}^{1} \dot{h}_{i}\left(V, \theta_{0}\right) d W(\rho)+\rho \int_{0}^{1} \nabla \dot{h}_{i}\left(V, \theta_{0}\right)
\end{aligned}
$$


for $i, j=1,2$, then we have

$$
\begin{aligned}
\frac{n^{\left(1+\beta_{0}\right) / 2}}{\log \sqrt{n}}\left(\hat{\alpha}_{n}-\alpha_{0}\right) & =-\alpha_{0} n^{\left(1+\beta_{0}\right) / 2}\left(\hat{\beta}_{n}-\beta_{0}\right)+O_{p}\left((\log \sqrt{n})^{-1}\right) \\
& \rightarrow_{d}\left(M_{22}-\frac{M_{12}^{2}}{M_{11}}\right)^{-1}\left(N_{2}(\rho)-\frac{M_{12} N_{1}(\rho)}{M_{11}}\right)
\end{aligned}
$$

as $n \rightarrow \infty$.

The logistic function $f$ given in (15) does not satisfy our conditions in any of Theorems 4.1 - 4.3. Therefore, we may not directly apply them to obtain the asymptotics in this case. However, if we write $f=f_{1}+f_{2}$, where

$$
\begin{aligned}
f_{1}(x, \alpha) & =\alpha 1\{x \geq 0\} \\
f_{2}(x, \alpha, \beta) & =\alpha\left(\frac{e^{\beta x}}{1+e^{\beta x}} 1\{x<0\}-\frac{1}{1+e^{\beta x}} 1\{x \geq 0\}\right)
\end{aligned}
$$

and define $f_{2}^{*}(x, \beta)=f_{2}\left(x, \alpha_{0}, \beta\right)$, then the NLS estimators $\hat{\alpha}_{n}$ and $\hat{\beta}_{n}$ from the regression with regression function $f$ have the same limiting distributions as the NLS estimators from the two separate regressions with regression functions $f_{1}$ and $f_{2}^{*}$ only. This is shown in Chang et al. (2005).

The regression functions $f_{1}$ and $f_{2}^{*}$ satisfy, respectively, the conditions in Theorems 4.2 and 4.1. We may readily deduce that

$$
\sqrt{n}\left(\hat{\alpha}_{n}-\alpha_{0}\right) \rightarrow_{d}\left(\int_{0}^{1} 1\{V \geq 0\}\right)^{-1}\left(\int_{0}^{1} 1\{V \geq 0\} d W(\rho)-\rho L(1,0)\right)
$$

and

$$
\sqrt[4]{n}\left(\hat{\beta}_{n}-\beta_{0}\right) \rightarrow{ }_{d} \mathbb{M N}\left(0,\left[\frac{\alpha_{0}^{2}\left(\pi^{2}-6\right)}{18 \beta_{0}^{3}} L(1,0)\right]^{-1}\right)
$$

as $n \rightarrow \infty$. The limiting distribution of the NLS estimator $\hat{\alpha}_{n}$ of the level parameter is affected by the presence of endogeneity, and given as a function of $\rho$. However, the distribution of the NLS estimator $\hat{\beta}_{n}$ of the transition parameter is independent of $\rho$ in the limit, and hence, is asymptotically invariant with respect to the endogeneity parameter $\rho$.

\section{Simulations}

In this section, we conduct simulations to convey the finite sample performances of the NLS estimators in the presence of endogeneity, and in particular they are compared to those obtained under no endogeneity. For our simulations, we consider the nonlinear regression model in (1) with the regression functions given by the power function and logistic function, specified respectively in (14) and (15). The regressor $\left(x_{t}\right)$ and the regression error $\left(\varepsilon_{t}\right)$ are allowed to be contemporaneously correlated and generated as in (2) and (3). To see how the varying degrees of endogeneity affect the finite sample performances of the NLS estimators and their $t$-statistics, we try three different values of the parameter $\rho$ which measures the 
fraction of the endogenous component of the regression error: $\rho=0,0.5$, and 1 . The innovations $\left(u_{t}\right)$ and $\left(v_{t}\right)$ are drawn from independent $\mathbb{N}\left(0, \sigma^{2}\right)$ with $\sigma=0.1$. The true parameter values of the level and transition parameters are set respectively at $\alpha_{0}=1, \beta_{0}=3$ for the regressions with the logistic regression function, and at $\alpha_{0}=1, \beta_{0}=0.5$ and 2 for the regressions with the power regression function. For the power function, two values for the true value of the power exponent $\beta_{0}$ are tried - one less than one and the other greater than one - to see how the behaviors of the NLS estimators change as the magnitude of the exponent $\beta_{0}$ changes, especially around the unity. Samples of sizes $n=200,500$ are considered and each simulation is run for 10,000 times.

The finite sample performances of the NLS estimators are presented via a set of figures with the estimated densities of the centered NLS estimators and the corresponding $t$-statistics. ${ }^{10}$ Figures $1-6$ present the results from the regression with power regression function. Figures $1-3$ provide the results for the case with $\beta_{0}=0.5$. Figures 1 and 2 present the estimated densities of the centered NLS estimators computed with the three different values of $\rho$ for the cases with $n=200$ and $n=500$, respectively. The densities of both unscaled and scaled centered NLS estimators are provided respectively in the left and right hand side columns of each figure. The scaling factors are computed according to the convergence rates derived in (16). Panels in each column represent the density estimates from using three different values of $\rho=0,0.5,1$. Figure 3 provides the estimated densities for the $t$-statistics for the sample sizes $n=200,500$. Figures $4-6$ provide the results for the case with $\beta_{0}=2$ in the same format as in Figures $1-3$. Figures $7-9$ present the results from the regressions with logistic regression function. The results are presented in the same format as in the cases with the power regression function given in Figures $1-3$. The scaling factors for the NLS estimators in this case are taken from the convergence rates given in (17) and (18).

The simulation results largely corroborate the limit theories derived in (16) - (18) earlier in Section 5. As expected from (16), the limit distributions of the NLS estimators for both parameters $\alpha$ and $\beta$ in the power function are affected by the presence of the endogeneity. When there is no endogeneity with $\rho=0$, the limit distributions of both NLS estimators $\hat{\alpha}_{n}$ and $\hat{\beta}_{n}$ become mixed normal and the their $t$-statistics have standard normal limit distribution. These can be seen from the panels in the first row of each figures. As $\rho$ increases, however, the distributions of the NLS estimators and $t$-statistics for both parameters become more nonstandard and nonnormal. This is true for the cases with both true values $\beta_{0}=0.5,2$ of the power exponent, although the density estimates appear to be more nonstandard and depart more from the normality when the true value of the power exponent $\beta_{0}$ is less than unity. The dependence on the degree of endogeneity $\rho$ of the distributions of the NLS estimators and their $t$-ratios does not vanish as the sample size increases, and indeed, the nonstandardness and nonnormality of the distributions persist.

The finite sample performance of the NLS estimators from the logistic regression is also as predicted by the limit theories given in (17) and (18). The estimated densities for the NLS estimators $\hat{\alpha}_{n}$ and $\hat{\beta}_{n}$ of $\alpha$ and $\beta$ indicate that the NLS estimator $\hat{\alpha}_{n}$ converges faster than $\hat{\beta}_{n}$. They also show that the distributions of the level parameter estimator $\hat{\alpha}_{n}$ depends

\footnotetext{
${ }^{10}$ The densities are estimated via usual kernel method using normal kernel function.
} 
on the degree $\rho$ of endogeneity present in the model, and that their dependence on $\rho$ does not vanish even with larger sample sizes. The distributions of the transition parameter estimator $\hat{\beta}_{n}$, however, do not depend upon the magnitude of $\rho$. In the smaller samples with $n=200$, they seem to have some biases when there is strong endogeneity with $\rho=1$, but they disappear as the sample size increases. The distributions of the $t$-statistics also corroborate our limit theory. The estimated densities of the $t$-ratios for the level parameter $\alpha$ are clearly nonnormal when $\rho \neq 0$, and the departure from the normality becomes much more pronounced as $\rho$ increases. This continues to be the case even when the sample size gets large. On the other hand, the estimated densities of the $t$-ratios for the transition parameter $\beta$ are quite close to the standard normal density which is also drawn in the same panel for comparison. Clearly, they approach closer to the limit standard normal density as the sample size increases.

\section{Conclusion}

In this article, we develop the asymptotic theory for the NLS estimators in the nonlinear regressions with integrated regressors that are contemporaneously correlated with the regression error. For the regression with regression function having asymptotically homogenous parameter derivatives, our results are largely comparable to those for the linear regressions with integrated regressors. The presence of endogeneity may only have a second-order effect on the NLS estimator. In particular, the NLS estimator has exactly the same convergence rate as in the cases without endogeneity. Only the limit distribution of the NLS estimator is affected by the presence of endogeneity. If the parameter derivative of regression function is integrable, however, the presence of endogeneity has no effect at all even on the limit distribution. In this case, the NLS estimator has the same limit distribution as the one in regression without endogeneity. The mixed normality of limit distribution of the NLS estimator, in particular, continues to hold regardless of endogeneity.

Here we only consider simple prototypical models, where the regressor and regression error are driven by iid innovations. In particular, we do not allow for general models generated by serially correlated innovations. The purpose of this simplification is to highlight and fully analyze the effects of the presence of endogeneity, and to establish the asymptotic results that are applicable for a wide class of regressions with various types of regression functions. Indeed, some of our results can be readily extended to more general models. Not all of our results, of course, hold exactly as presented in the article for more general models. However, we believe that our main results for the prototypical models, at least qualitatively, continue to hold for models that are much more general and realistic than the simple models considered in the article. We leave as future research more rigorous developments of the asymptotic theory for general nonlinear models with integrated regressors in the presence of endogeneity. 


\section{Mathematical Proofs}

\subsection{Useful Lemmas}

Lemma A1 Let $T$ be a transformation on $\mathbb{R}$.

(a) If Assumptions 2.2 with $\nu=2$, and 2.3 hold, and if $T$ is locally Riemann-integrable and

$$
|T(x)|<c\left(1+|x|^{-1-\epsilon}\right)
$$

for some $c>0$ and $\epsilon>0$, then

$$
\frac{1}{\sqrt{n}} \sum_{t=1}^{n} T\left(x_{t}\right) \rightarrow_{d} L(1,0) \int_{-\infty}^{\infty} T(x) \mu(d x)
$$

as $n \rightarrow \infty$.

(b) If Assumptions 2.2 with $\nu=3$, and 2.3 hold, and if $T$ is bounded and

$$
\int_{-\infty}^{\infty}|x|^{1 / 2+\epsilon}|T(x)| \mu(d x)<\infty
$$

for some $\epsilon>0$, then we have

$$
\begin{aligned}
& \frac{1}{\sqrt[4]{n}} \sum_{t=1}^{n} T\left(x_{t}\right) v_{t} \rightarrow_{d} \mathbb{M N}\left(0, L(1,0) \int_{-\infty}^{\infty} T^{2}(x) \mu(d x)\right) \\
& \frac{1}{\sqrt[4]{n}} \sum_{t=1}^{n} T\left(x_{t}\right) u_{t} \rightarrow_{d} \mathbb{M N}\left(0, L(1,0) \int_{-\infty}^{\infty} T^{2}(x) \mu(d x)\right)
\end{aligned}
$$

independently of each other, as $n \rightarrow \infty$.

Proof of Lemma A1 For part (a), see Theorems 2.1, Chapter IV, in Borodin and Ibragimov (1995). To prove (19) in part (b), we let

$$
f(x, y)=T(y)(y-x)
$$

and apply Theorems 1.1 and 1.3 of Borodin (1986). Note that we have $f(x, x+y)=T(x+$ $y) y$. The integrability of $T$ follows immediately from the condition $\int_{-\infty}^{\infty}|x|^{1 / 2+\epsilon}|T(x)| \mu(d x)<$ $\infty$. We also have the square integrability of $T$ with respect to both the Lebesgue and counting measures. Clearly, with respect to the counting measure, the square integrability of $T$ is implied by the integrability $T$. Moreover, being bounded, the square integrability $T$ with respect to Lebesgue measure also follows from the integrability of $T$. We therefore have $\int_{-\infty}^{\infty} T^{2}(x) \mu(d x)<\infty$ under the given conditions.

We first check if the conditions (1.1), (1.2), (1.7) and (1.8) in Borodin (1986) are met. We have

$$
\begin{aligned}
\int \mathbb{E} f^{2}\left(x, x+v_{t}\right) \mu(d x) & =\int_{-\infty}^{\infty} \int_{-\infty}^{\infty} f^{2}(x, x+y) p(y) \mu(d y) \mu(d x) \\
& =\int_{-\infty}^{\infty} \int_{-\infty}^{\infty} T^{2}(x+y) y^{2} p(y) \mu(d y) \mu(d x) \\
& =\left(\int_{-\infty}^{\infty} T^{2}(x) \mu(d x)\right)\left(\int_{-\infty}^{\infty} y^{2} p(y) \mu(d y)\right)<\infty
\end{aligned}
$$


as required. Moreover,

$$
\begin{aligned}
\int_{-\infty}^{\infty} \mathbb{E}\left|v_{t}\right|^{1 / 2+\epsilon}\left|f\left(x, x+v_{t}\right)\right| \mu(d x) & =\int_{-\infty}^{\infty} \int_{-\infty}^{\infty}|f(x, x+y)||y|^{1 / 2+\epsilon} p(y) \mu(d y) \mu(d x) \\
& =\int_{-\infty}^{\infty} \int_{-\infty}^{\infty}|T(x+y)||y|^{3 / 2+\epsilon} p(y) \mu(d y) \mu(d x) \\
& =\left(\int_{-\infty}^{\infty}|T(x)| \mu(d x)\right)\left(\int_{-\infty}^{\infty}|y|^{2 / 3+\epsilon} p(y) \mu(d y)\right) \\
& <\infty
\end{aligned}
$$

and

$$
\begin{aligned}
\int_{-\infty}^{\infty} \mathbb{E}|x|^{1 / 2+\epsilon}\left|f\left(x, x+v_{t}\right)\right| \mu(d x) & =\int_{-\infty}^{\infty} \int_{-\infty}^{\infty}|x|^{1 / 2+\epsilon}|f(x, x+y)| p(y) \mu(d y) \mu(d x) \\
& =\int_{-\infty}^{\infty} \int_{-\infty}^{\infty}|x|^{1 / 2+\epsilon}|T(x+y)||y| p(y) \mu(d y) \mu(d x) \\
& \leq \int_{-\infty}^{\infty} \int_{-\infty}^{\infty}\left(|x|^{1 / 2+\epsilon}+|y|^{1 / 2+\epsilon}\right)|T(x)||y| p(y) \mu(d y) \mu(d x) \\
& =\left(\int_{-\infty}^{\infty}|x|^{1 / 2+\epsilon}|T(x)| \mu(d x)\right)\left(\int_{-\infty}^{\infty}|y|^{3 / 2+\epsilon} p(y) \mu(d y)\right) \\
& <\infty
\end{aligned}
$$

as was to be shown.

We now let $h, \nu, \rho$ and $b$ be defined as in Borodin (1986). Then it follows that

$$
\begin{aligned}
h(x) & =\mathbb{E} f\left(x, x+v_{t}\right) \\
& =\int_{-\infty}^{\infty} f(x, x+y) p(y) \mu(d y) \\
& =\int_{-\infty}^{\infty} T(x+y) y p(y) \mu(d y)
\end{aligned}
$$

and therefore, $\int_{-\infty}^{\infty} h(x) \mu(d x)=0$. We also have

$$
\begin{aligned}
\nu(x) & =\int_{-\infty}^{\infty} e^{-i x y} h(y) \mu(d y) \\
& =\int_{-\infty}^{\infty} e^{-i x y} \int_{-\infty}^{\infty} T(y+z) z p(z) \mu(d z) \mu(d y) \\
& =\int_{-\infty}^{\infty} e^{-i x(y-z)} \int_{-\infty}^{\infty} T(y) z p(z) \mu(d z) \mu(d y) \\
& =\left(\int_{-\infty}^{\infty} e^{-i x y} T(y) \mu(d y)\right)\left(\int_{-\infty}^{\infty} e^{i x z} z p(z) \mu(d z)\right)
\end{aligned}
$$

so that

$$
|\nu(x)| \leq\left(\int_{-\infty}^{\infty}|T(y)| \mu(d y)\right)\left(\int_{-\infty}^{\infty}|z| p(z) \mu(d z)\right)
$$


i.e., $\nu$ is bounded by a constant, and

$$
\begin{aligned}
\rho(x) & =\int_{-\infty}^{\infty} e^{i x y} \mathbb{E}\left(e^{i y v_{t}} f\left(y, y+v_{t}\right)\right) \mu(d y) \\
& =\int_{-\infty}^{\infty} e^{i x y} \int e^{i y z} f(y, y+z) p(z) \mu(d z) \mu(d y) \\
& =\int_{-\infty}^{\infty} e^{i x y} \int_{-\infty}^{\infty} e^{i y z} T(y+z) z p(z) \mu(d z) \mu(d y) \\
& =\int_{-\infty}^{\infty} e^{i x(y-z)} \int_{-\infty}^{\infty} e^{i y z} T(y) z p(z) \mu(d z) \mu(d y) \\
& =\left(\int_{-\infty}^{\infty} e^{i x y} T(y) \mu(d y)\right)\left(\int_{-\infty}^{\infty} z p(z) \mu(d z)\right)=0
\end{aligned}
$$

Finally,

$$
\begin{aligned}
b & =\int_{-\infty}^{\infty} \mathbb{E} f^{2}\left(x, x+v_{t}\right) \mu(d x) \\
& =\int_{-\infty}^{\infty} \int_{-\infty}^{\infty} f^{2}(x, x+y) p(y) \mu(d y) \mu(d x) \\
& =\int_{-\infty}^{\infty} \int_{-\infty}^{\infty} T^{2}(x+y) y^{2} p(y) \mu(d y) \mu(d x) \\
& =\int_{-\infty}^{\infty} T^{2}(x) \mu(d x)
\end{aligned}
$$

The result in (19) of part (b) now follows immediately from Borodin (1986). The result in (20) of part (b) is a special case of Theorem 3.2 in PP, and the independence of limit distributions in (19) and (20) follows immediately from the independence of $\left(u_{t}\right)$ and $\left(v_{t}\right)$.

Lemma A2 If Assumptions 2.2 with $\nu=3$ and 2.3 hold, then

$$
\begin{aligned}
& \frac{1}{\sqrt{n}} \sum_{t=1}^{n}\left(1\left\{x_{t} \geq 0\right\}-1\left\{x_{t-1} \geq 0\right\}\right) v_{t} \rightarrow_{d} L(1,0) \\
& \frac{1}{\sqrt{n}} \sum_{t=1}^{n}\left(1\left\{x_{t}<0\right\}-1\left\{x_{t-1}<0\right\}\right) v_{t} \rightarrow_{d}-L(1,0)
\end{aligned}
$$

as $n \rightarrow \infty$.

Proof of Lemma A2 Let

$$
f(x, y)=(1\{y \geq 0\}-1\{x \geq 0\})(y-x)
$$

note that

$$
\begin{aligned}
f\left(x, x+v_{t}\right) & =\left(1\left\{x+v_{t} \geq 0\right\}-1\{x \geq 0\}\right) v_{t} \\
& =\left(1\left\{x+v_{t} \geq 0\right\} 1\{x<0\}-1\left\{x+v_{t}<0\right\} 1\{x \geq 0\}\right) v_{t} \\
& =\left(1\left\{v_{t} \geq-x>0\right\}-1\left\{v_{t}<-x \leq 0\right\}\right) v_{t}
\end{aligned}
$$


As in the proof of Lemma A1, we first check the conditions for Theorems 1.1 and 1.3 of Borodin (1986).

We have

$$
\begin{aligned}
& \int_{-\infty}^{\infty} \mathbb{E} f^{2}\left(x, x+v_{t}\right) \mu(d x) \\
& =\int_{-\infty}^{\infty} \int_{-\infty}^{\infty}(1\{y \geq-x>0\}+1\{y<-x \leq 0\}) y^{2} p(y) \mu(d y) \mu(d x) \\
& =\left(\int_{x<0} \int_{y \geq-x}+\int_{x \geq 0} \int_{y<-x}\right) y^{2} p(y) \mu(d y) \mu(d x)<\infty
\end{aligned}
$$

Furthermore,

$$
\begin{aligned}
& \int_{-\infty}^{\infty} \mathbb{E}\left|v_{t}\right|^{1 / 2+\epsilon}\left|f\left(x, x+v_{t}\right)\right| \mu(d x) \\
& \leq \int_{-\infty}^{\infty} \int_{-\infty}^{\infty}(1\{y \geq-x>0\}+1\{y<-x \leq 0\})|y|^{3 / 2+\epsilon} p(y) \mu(d y) \mu(d x) \\
& =\left(\int_{x<0} \int_{y \geq-x}+\int_{x \geq 0} \int_{y<-x}\right)|y|^{3 / 2+\epsilon} p(y) \mu(d y) \mu(d x)<\infty
\end{aligned}
$$

and

$$
\begin{aligned}
& \int_{-\infty}^{\infty} \mathbb{E}|x|^{1 / 2+\epsilon}\left|f\left(x, x+v_{t}\right)\right| \mu(d x) \\
& \quad \leq \int_{-\infty}^{\infty}|x|^{1 / 2+\epsilon} \int_{-\infty}^{\infty}(1\{y \geq-x>0\}+1\{y<-x \leq 0\})|y| p(y) \mu(d y) \mu(d x) \\
& =\left(\int_{x<0}(-x)^{1 / 2+\epsilon} \int_{y \geq-x}+\int_{x \geq 0} x^{1 / 2+\epsilon} \int_{y<-x}\right)|y| p(y) \mu(d y) \mu(d x)<\infty
\end{aligned}
$$

as was to be shown.

Now we let

$$
h(x)=\mathbb{E} f\left(x, x+v_{t}\right)
$$

Then we have

$$
\begin{aligned}
\mathbb{E} f\left(x, x+v_{t}\right) & =-1\{x \geq 0\} \int_{y<-x} y p(y) \mu(d y)+1\{x<0\} \int_{y \geq-x} y p(y) \mu(d y) \\
& =\int_{y \geq-x} y p(y) \mu(d y)
\end{aligned}
$$

since $\int_{-\infty}^{\infty} y p(y) \mu(d y)=0$. Therefore, if follows from Theorem 1.1 and 1.4 of Borodin (1986) that

$$
\begin{aligned}
\frac{1}{\sqrt{n}} \sum_{t=1}^{n} f\left(x_{t-1}, x_{t}\right) & \rightarrow{ }_{d} L(1,0) \int_{-\infty}^{\infty} h(x) \mu(d x) \\
& =L(1,0) \int_{-\infty}^{\infty} \int_{y \geq-x} y p(y) \mu(d y) \mu(d x)
\end{aligned}
$$


as $n \rightarrow \infty$.

To finish the proof of the first part of the stated results, we note that

$$
\begin{aligned}
\int_{-\infty}^{\infty} & \int_{y \geq-x} y p(y) \mu(d y) \mu(d x) \\
& =\int_{-\infty}^{\infty} \int_{y \geq-x} 1\{x \geq 0\} y p(y) \mu(d y) \mu(d x)+\int_{-\infty}^{\infty} \int_{y \geq-x} 1\{x<0\} y p(y) \mu(d y) \mu(d x) \\
& =-\int_{-\infty}^{\infty} \int_{y<-x} 1\{x \geq 0\} y p(y) \mu(d y) \mu(d x)+\int_{-\infty}^{\infty} \int_{y \geq-x} 1\{x<0\} y p(y) \mu(d y) \mu(d x) \\
& =-\int_{y>0} \int_{-y \leq x<0} y p(y) \mu(d x) \mu(d y)+\int_{y<0} \int_{0 \leq x<y} y p(y) \mu(d x) \mu(d y) \\
& =\int_{y>0} y^{2} p(y) \mu(d y)+\int_{y<0} y^{2} p(y) \mu(d y) \\
& =\int_{-\infty}^{\infty} y^{2} p(y) \mu(d y)=\sigma_{v}^{2},
\end{aligned}
$$

and that we set $\sigma_{v}^{2}=1$. We thus have shown the first part of the stated results. The proof for the second part of the stated results is completely analogous and omitted.

Lemma A3 Let $T$ be a transformation on $\mathbb{R}$ such that

(a) $|T(x)| \leq c|x|^{a}$ for all $\mathbb{R} \backslash\{0\}$ in a neighborhood of origin for some constants $a>-1$ and $c>0$, and

(b) $T(x)$ is differentiable with continuous derivative for all $\mathbb{R} \backslash\{0\}$ such that $\left|T^{\prime}(x)\right| \leq$ $c|x|^{b}$ for some constants $b$ and $c>0$ in a neighborhood of the origin.

Then we have

$$
\int_{0}^{1} T\left(V_{n}(r)\right) d r \rightarrow_{d} \int_{0}^{1} T(V(r)) d r
$$

as $n \rightarrow \infty$.

Proof of Lemma A3 The stated result follows directly from the proof of Theorem 3.2 in Park (2003).

Lemma A4 All the results in Lemmas A1, A2 and A3 hold jointly, and jointly with the invariance principle in (6).

Proof of Lemma A4 All our results in Lemmas A1, A2 and A3 are based on Borodin (1986) and PP. Both of them use the Skorokhod embedding, which allows us to construct the partial sum process $\left(U_{n}, V_{n}\right)$ in (6) from $\left(u_{t}^{n}\right)$ and $\left(v_{t}^{n}\right)$ such that $\left(u_{t}^{n}\right)=_{d}\left(u_{t}\right)$ and $\left(v_{t}^{n}\right)={ }_{d}\left(v_{t}\right)$ and that $\left(U_{n}, V_{n}\right)$ converges almost surely to the limit Brownian motion $(U, V)$. In this setting, we may deduce the results in part (a) of Lemma A1, Lemma A2 and Lemma A3 in the mode of almost sure convergence, as in Borodin (1986) and PP. It is therefore clear that they hold jointly with the distributional convergence results in part (b) of Lemma A1. Consequently, all the results in Lemmas A1, A2 and A3 hold jointly in the distributional sense. 


\subsection{Proofs of Theorems}

Proof of Lemma 3.2 For the proof of part (a), we write

$$
\frac{1}{\sqrt{n}} \sum_{t=1}^{n} T\left(\frac{x_{t}}{\sqrt{n}}\right) v_{t}=\frac{1}{\sqrt{n}} \sum_{t=1}^{n} T\left(\frac{x_{t-1}}{\sqrt{n}}\right) v_{t}+\frac{1}{\sqrt{n}} \sum_{t=1}^{n}\left[T\left(\frac{x_{t}}{\sqrt{n}}\right)-T\left(\frac{x_{t-1}}{\sqrt{n}}\right)\right] v_{t}
$$

It follows immediately from PP that

$$
\frac{1}{\sqrt{n}} \sum_{t=1}^{n} T\left(\frac{x_{t-1}}{\sqrt{n}}\right) v_{t} \rightarrow_{d} \int_{0}^{1} T(V(r)) d V(r)
$$

as $n \rightarrow \infty$. Next, we write

$$
\frac{1}{\sqrt{n}} \sum_{t=1}^{n}\left[T\left(\frac{x_{t}}{\sqrt{n}}\right)-T\left(\frac{x_{t-1}}{\sqrt{n}}\right)\right] v_{t}=A_{n}+B_{n}+C_{n}+D_{n}
$$

where

$$
\begin{aligned}
& A_{n}=\frac{1}{\sqrt{n}} \sum_{t=1}^{n}\left[T\left(\frac{x_{t}}{\sqrt{n}}\right)-T\left(\frac{x_{t-1}}{\sqrt{n}}\right)\right] 1\left\{x_{t} \geq 0\right\} 1\left\{x_{t-1} \geq 0\right\} v_{t} \\
& B_{n}=\frac{1}{\sqrt{n}} \sum_{t=1}^{n}\left[T\left(\frac{x_{t}}{\sqrt{n}}\right)-T\left(\frac{x_{t-1}}{\sqrt{n}}\right)\right] 1\left\{x_{t} \geq 0\right\} 1\left\{x_{t-1}<0\right\} v_{t} \\
& C_{n}=\frac{1}{\sqrt{n}} \sum_{t=1}^{n}\left[T\left(\frac{x_{t}}{\sqrt{n}}\right)-T\left(\frac{x_{t-1}}{\sqrt{n}}\right)\right] 1\left\{x_{t}<0\right\} 1\left\{x_{t-1} \geq 0\right\} v_{t} \\
& D_{n}=\frac{1}{\sqrt{n}} \sum_{t=1}^{n}\left[T\left(\frac{x_{t}}{\sqrt{n}}\right)-T\left(\frac{x_{t-1}}{\sqrt{n}}\right)\right] 1\left\{x_{t}<0\right\} 1\left\{x_{t-1}<0\right\} v_{t}
\end{aligned}
$$

Whenever $x_{t-1}<0$ and $x_{t} \geq 0$, we have

$$
v_{t}>0
$$

Therefore, if we define

$$
\begin{aligned}
& M_{n}=\frac{1}{\sqrt{n}} \sum_{t=1}^{n} 1\left\{x_{t} \geq 0\right\} 1\left\{x_{t-1}<0\right\} v_{t} \\
& N_{n}=\sup _{1 \leq t \leq n}\left|T\left(\frac{x_{t}}{\sqrt{n}}\right)-T\left(\frac{x_{t-1}}{\sqrt{n}}\right)\right| 1\left\{x_{t} \geq 0\right\} 1\left\{x_{t-1}<0\right\}
\end{aligned}
$$

then it follows that

$$
B_{n} \leq M_{n} N_{n}
$$

Since $T$ is assumed to be locally bounded, we have $N_{n}=O_{p}(1)$. Moreover, it follows from the proof of Lemma A2 that $M_{n}=O_{p}(1)$. We therefore have $B_{n}=O_{p}(1)$. It is quite obvious that we may use the same argument and show that $C_{n}=O_{p}(1)$. 
Now we consider $A_{n}$ and $D_{n}$. When $x_{t}>0$ and $x_{t-1}>0$, we have

$$
T\left(\frac{x_{t}}{\sqrt{n}}\right)-T\left(\frac{x_{t-1}}{\sqrt{n}}\right)=\nabla T\left(\frac{x_{t-1}}{\sqrt{n}}+\frac{w_{t}}{\sqrt{n}}\right) \frac{v_{t}}{\sqrt{n}}
$$

where $\left(w_{t}\right)$ is a random sequence such that $\left|w_{t}\right| \leq\left|v_{t}\right|$. It follows that

$$
\begin{aligned}
A_{n}= & \frac{1}{\sqrt{n}} \sum_{t=1}^{n}\left[T\left(\frac{x_{t}}{\sqrt{n}}\right)-T\left(\frac{x_{t-1}}{\sqrt{n}}\right)\right] 1\left\{x_{t} \geq 0\right\} 1\left\{x_{t-1} \geq 0\right\} v_{t} \\
= & \frac{1}{n} \sum_{t=1}^{n} \nabla T\left(\frac{x_{t-1}}{\sqrt{n}}+\frac{w_{t}}{\sqrt{n}}\right) 1\left\{x_{t} \geq 0\right\} 1\left\{x_{t-1} \geq 0\right\} v_{t}^{2} \\
= & \frac{1}{n} \sum_{t=1}^{n} \nabla T\left(\frac{x_{t-1}}{\sqrt{n}}+\frac{w_{t}}{\sqrt{n}}\right) 1\left\{x_{t} \geq 0\right\} 1\left\{x_{t-1} \geq 0\right\} \\
& +\frac{1}{n} \sum_{t=1}^{n} \nabla T\left(\frac{x_{t-1}}{\sqrt{n}}+\frac{w_{t}}{\sqrt{n}}\right) 1\left\{x_{t} \geq 0\right\} 1\left\{x_{t-1} \geq 0\right\}\left(v_{t}^{2}-1\right)
\end{aligned}
$$

Under the given conditions, we have

$$
\frac{1}{n} \sum_{t=1}^{n} \nabla T\left(\frac{x_{t-1}}{\sqrt{n}}+\frac{w_{t}}{\sqrt{n}}\right) 1\left\{x_{t} \geq 0\right\} 1\left\{x_{t-1} \geq 0\right\} \rightarrow_{d} \int_{0}^{1} \nabla T(V(r)) 1\{V(r) \geq 0\} d r
$$

due to Lemma A3. Moreover,

$$
\frac{1}{\sqrt{n}} \sum_{t=1}^{n} \nabla T\left(\frac{x_{t-1}}{\sqrt{n}}\right) 1\left\{x_{t} \geq 0\right\} 1\left\{x_{t-1} \geq 0\right\}\left(v_{t}^{2}-1\right)=O_{p}(1)
$$

and we may easily show that

$$
\frac{1}{n} \sum_{t=1}^{n}\left(\nabla T\left(\frac{x_{t-1}}{\sqrt{n}}+\frac{w_{t}}{\sqrt{n}}\right)-\nabla T\left(\frac{x_{t-1}}{\sqrt{n}}\right)\right) 1\left\{x_{t} \geq 0\right\} 1\left\{x_{t-1} \geq 0\right\}\left(v_{t}^{2}-1\right)=o_{p}(1)
$$

using Cauchy-Schwarz and Lemma A3. Note that

$$
\max _{1 \leq t \leq n} \frac{\left|w_{t}\right|}{\sqrt{n}} \leq \max _{1 \leq t \leq n} \frac{\left|v_{t}\right|}{\sqrt{n}} \rightarrow{ }_{p} 0
$$

We thus have shown that $A_{n}=O_{p}(1)$. We may similarly show that $D_{n}=O_{p}(1)$. The proof for part (a) is therefore complete.

For part (b), we first consider the case that $T$ has vanishing derivative on $\mathbb{R} \backslash\{0\}$. We may then assume without loss of generality that $T(x)=a 1\{x \geq 0\}+b 1\{x<0\}$ for some constants $a$ and $b$. Write

$$
\begin{aligned}
& \frac{1}{\sqrt{n}} \sum_{t=1}^{n} 1\left\{x_{t} \geq 0\right\} v_{t} \\
& =\frac{1}{\sqrt{n}} \sum_{t=1}^{n} 1\left\{x_{t-1} \geq 0\right\} v_{t}+\frac{1}{\sqrt{n}} \sum_{t=1}^{n}\left(1\left\{x_{t} \geq 0\right\}-1\left\{x_{t-1} \geq 0\right\}\right) v_{t}
\end{aligned}
$$


Then we may deduce from PP that

$$
\frac{1}{\sqrt{n}} \sum_{t=1}^{n} 1\left\{x_{t-1} \geq 0\right\} v_{t} \rightarrow{ }_{d} \int_{0}^{1} 1\{V(r) \geq 0\} d V(r)
$$

as $n \rightarrow \infty$, and the stated result follows from Lemma A2.

We now consider the case for which $T$ is continuous and has nonvanishing derivative. We have (21) - (23) as in the proof of part (a). We first show that

$$
B_{n}=o_{p}(1)
$$

Whenever $x_{t-1}<0$ and $x_{t} \geq 0$, we have

$$
\left|x_{t}\right|,\left|x_{t-1}\right| \leq\left|v_{t}\right|
$$

as well as $v_{t}>0$. If we let $N_{n}$ be defined as in (25), then we may therefore readily deduce that $N_{n}=o_{p}(1)$, since $T$ is continuous at the origin and

$$
\max _{1 \leq t \leq n} \frac{\left|v_{t}\right|}{\sqrt{n}} \rightarrow_{p} 0
$$

This establishes (28). It is quite obvious that we may deduce

$$
C_{n}=o_{p}(1)
$$

similarly as for $B_{n}$.

We may write $A_{n}$ as in (27) and obtain

$$
A_{n} \rightarrow \int_{0}^{1} \nabla T(V(r)) 1\{V(r) \geq 0\} d r
$$

as $n \rightarrow \infty$. Similarly, we may derive

$$
D_{n} \rightarrow_{d} \int_{0}^{1} \nabla T(V(r)) 1\{V(r)<0\} d r
$$

as $n \rightarrow \infty$. The stated result now follows from (22) and (28) - (31).

Proof of Lemma 3.5 The stated result follows immediately from part (b) of Lemma A1.

Proof of Lemma 3.6 From Definition 3.4 we have $F(\lambda x, \pi)=\kappa(\lambda, \pi) H(x, \pi)+R(x, \lambda, \pi)$, and this gives

$$
\frac{1}{\sqrt{n}} \kappa(\sqrt{n}, \pi)^{-1} \sum_{t=1}^{n} F\left(x_{t}, \pi\right) v_{t}=\frac{1}{\sqrt{n}} \sum_{t=1}^{n} H\left(\frac{x_{t}}{\sqrt{n}}, \pi\right) v_{t}+o_{p}(1)
$$


Note that $Q$ is regular and

$$
\frac{1}{\sqrt{n}} \sum_{t=1}^{n} Q\left(\frac{x_{t}}{\sqrt{n}}, \pi\right) v_{t}=O_{p}(1)
$$

due to Lemma $3.2(\mathrm{a})$, and $\left(\kappa^{-1} \varpi\right)(\lambda) \rightarrow 0$ as $\lambda \rightarrow \infty$. Note also that $\kappa(\cdot, \pi)=1$ when $H(\cdot, \pi)$ has vanishing derivative. The stated results now follow directly from Lemma 3.2 (b).

Proof of Theorem 4.1 Under the given conditions and our previous results in (8), (9) and Lemma 3.5, we may show that the NLS estimator $\hat{\theta}_{n}$ is asymptotically equivalent to the least squares estimator of $\theta$ in the linear regression

$$
y_{t}=\dot{f}\left(x_{t}, \theta_{0}\right)^{\prime} \theta+\varepsilon_{t}
$$

The stated result thus follows immediately. The proof is essentially identical to that of Theorem 5.1 in PP, except for our covariance asymptotics under endogeneity given in Lemma 3.5 .

Proof of Theorem 4.2 Once again, the proof is virtually the same as that of Theorem 5.2 in PP. We only need to replace their covariance asymptotics (11) by a mixture of our covariance asymptotics under endogeneity in Lemma 3.6 and theirs. In this case, we also have the asymptotic equivalence between the NLS estimator $\hat{\theta}_{n}$ and the least squares estimator of $\theta$ in the linear regression (32) defined in the proof of Theorem 4.1.

Proof of Thoerem 4.3 The arguments in the proofs of Theorem 4.1 and 4.2 also apply to the proof of Theorem 4.3, which is analogous to that of Theorem 5.3 in PP. The details are therefore omitted. 


\section{References}

Akonom, J. (1993). "Comportement asymptotique du temps d'occupation du processus des sommes partielles," Annales de l'Institut Henri Poincaré, 29, 57-81.

Borodin, A.N. (1986). "On the character of convergence to Brownian local time. II," Probability Theory and Related Fields 72, 251-277.

Borodin, A.N. and I.A. Ibragimov (1995). Limit Theorems for Functionals of Random Walks, Proceedings of the Steklov Institute of Mathematics, Vol 195, American Mathematical Society, Rhode Island.

Chang, Y., B. Jiang and J.Y. Park (2005). "Nonstationary logistic regression," Mimeograph, Department of Economics, Rice University.

Chang, Y. and J.Y. Park (2003). "Nonstationary index models," Journal of Econometrics, $114,73-106$.

Chang, Y., J.Y. Park and P.C.B. Phillips (2001). "Nonlinear econometric models with cointegrated and deterministically trending regressors," Econometrics Journal, 4, 136 .

de Jong, R. (2002). "Nonlinear regression with integrated regressors but without exogeneity," Mimeograph, Department of Economics, Michigan State University.

Jaganathan, P. (2008). "Limit theorems for functionals of sums that converge to fractional Brownian and stable motions," Cowles Foundation Discussion Paper No. 1649, Cowles Foundation for Research in Economics, Yale University.

Park, J.Y. (2003). "Strong approximations for nonlinear transformations of integrated time series," Mimeograph, Department of Economics, Rice University.

Park, J.Y. and P.C.B. Phillips (1999). "Asymptotics for nonlinear transformations of integrated time series", Econometric Theory, 15, 269-298.

Park, J.Y. and P.C.B. Phillips (2000). "Nonstationary binary choice", Econometrica, 68, 1249-1280.

Park, J.Y. and P.C.B. Phillips (2001). "Nonlinear regressions with integrated time series", Econometrica, 69 1452-1498. 

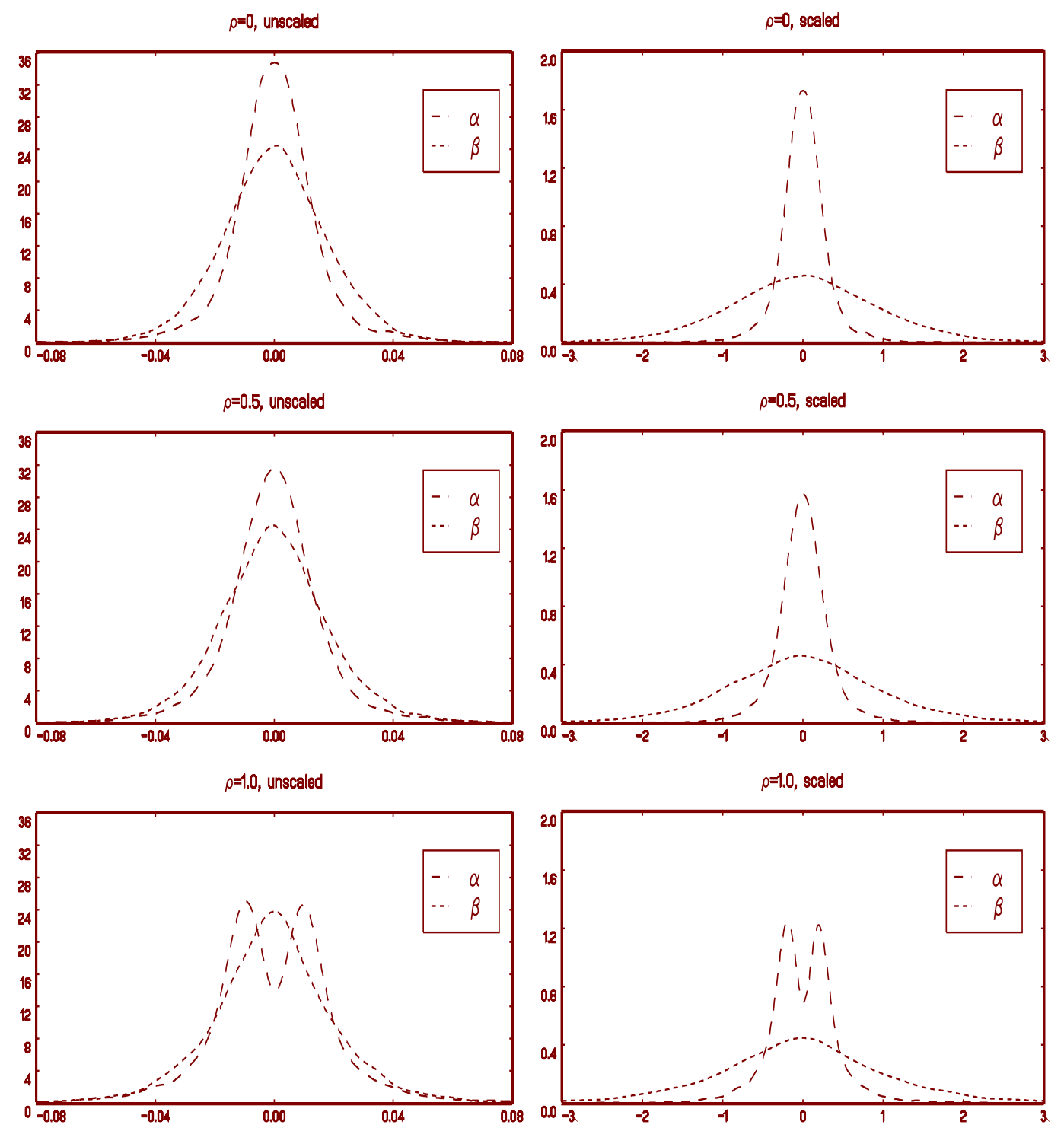

Figure 1: Densities of NLS Estimators, Power Function, $\beta_{0}=0.5, n=200$ 

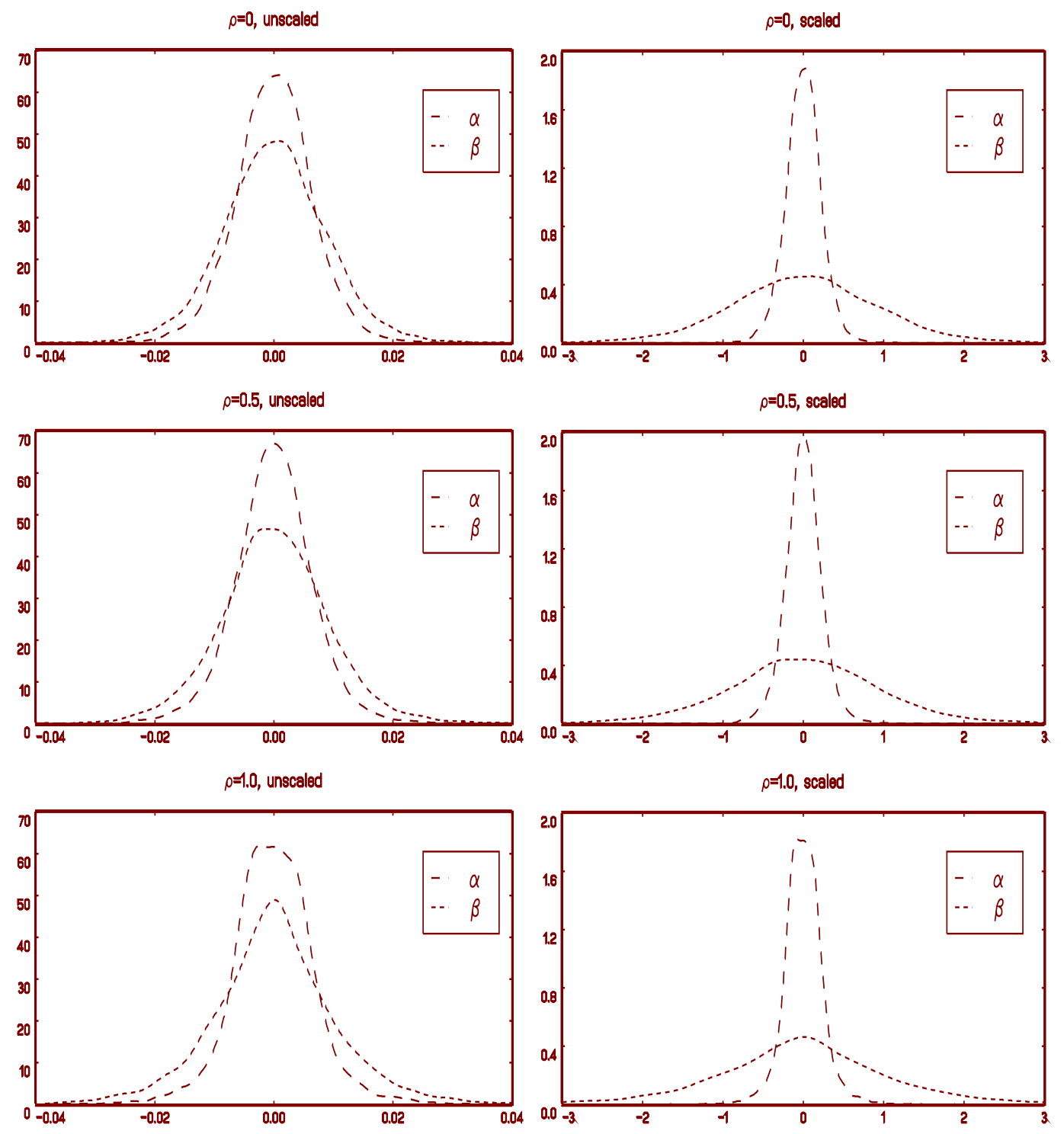

Figure 2: Densities of NLS Estimators, Power Function, $\beta_{0}=0.5, n=500$ 

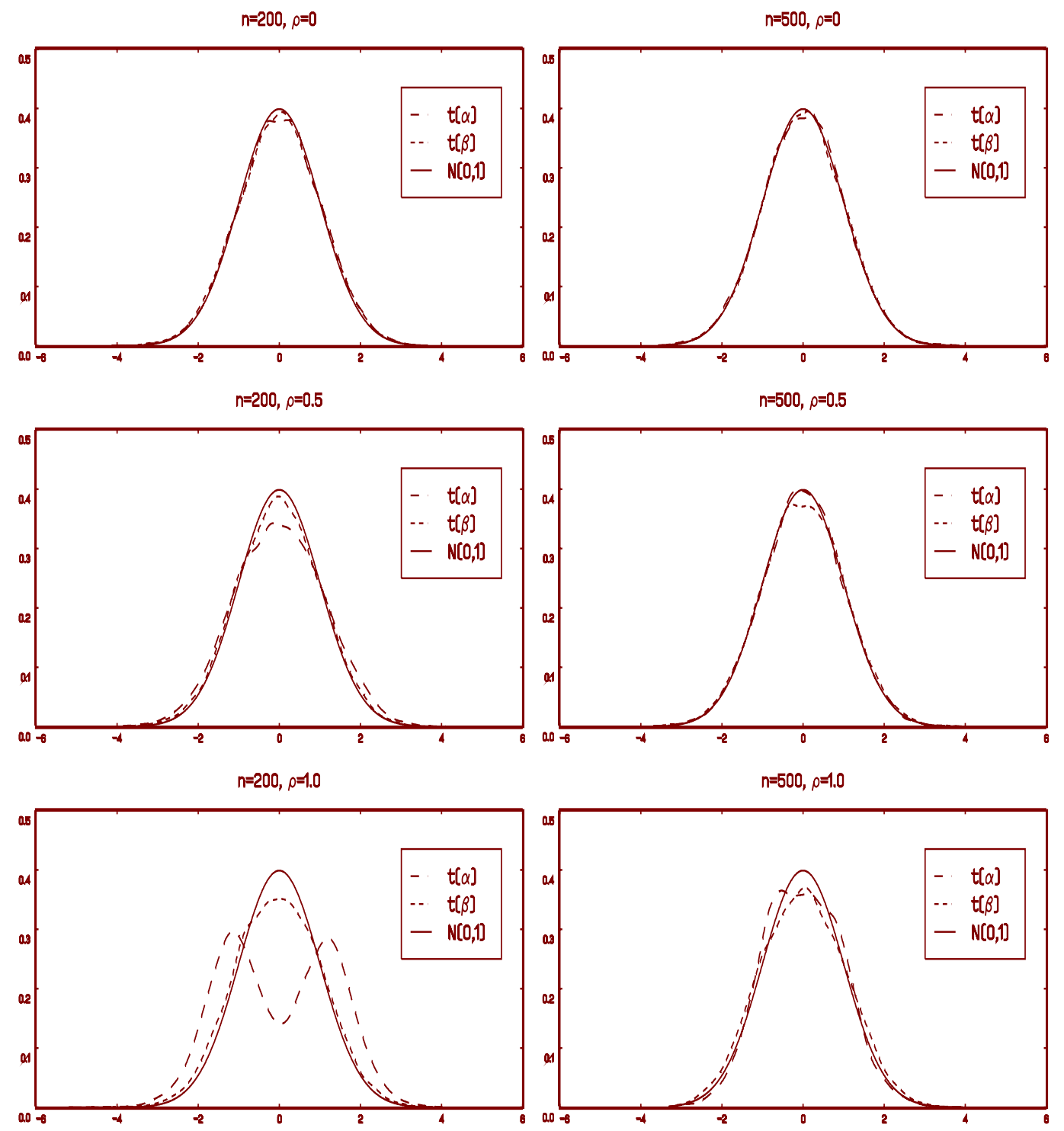

Figure 3: Densities of NLS $t$-ratios, Power Function, $\beta_{0}=0.5$ 

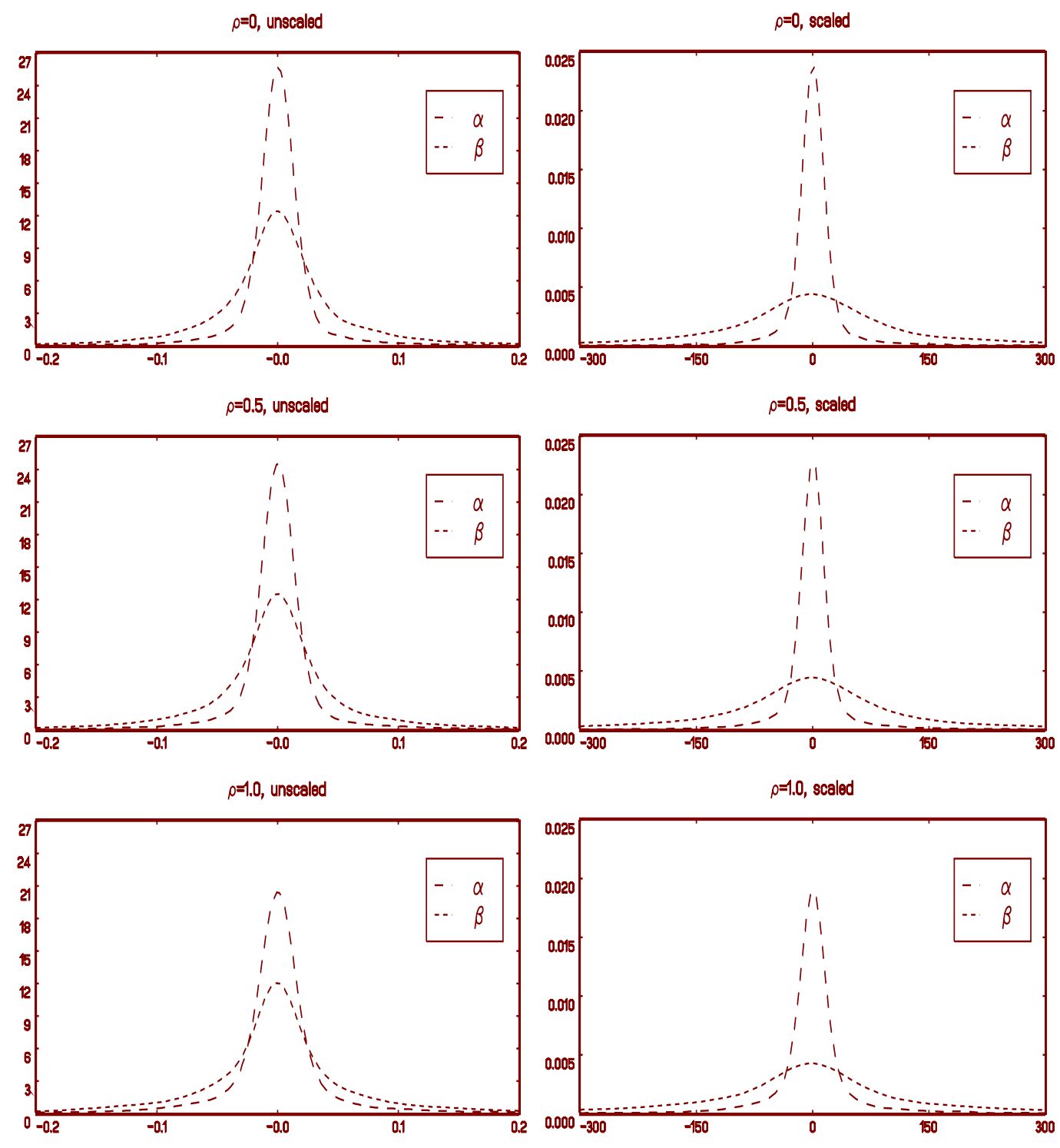

Figure 4: Densities of NLS Estimators, Power Function, $\beta_{0}=2, n=200$ 

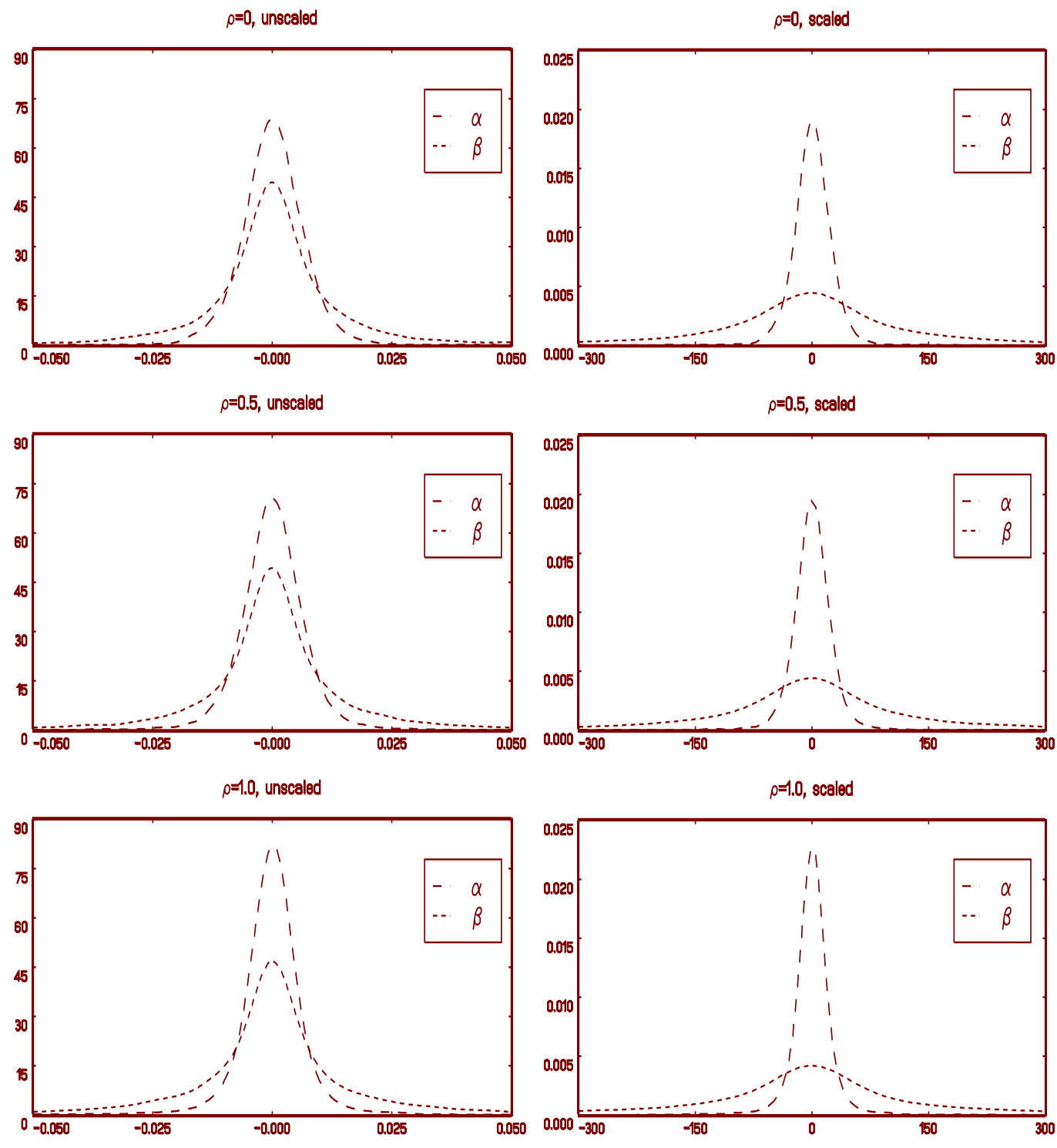

Figure 5: Densities of NLS Estimators, Power Function, $\beta_{0}=2, n=500$ 

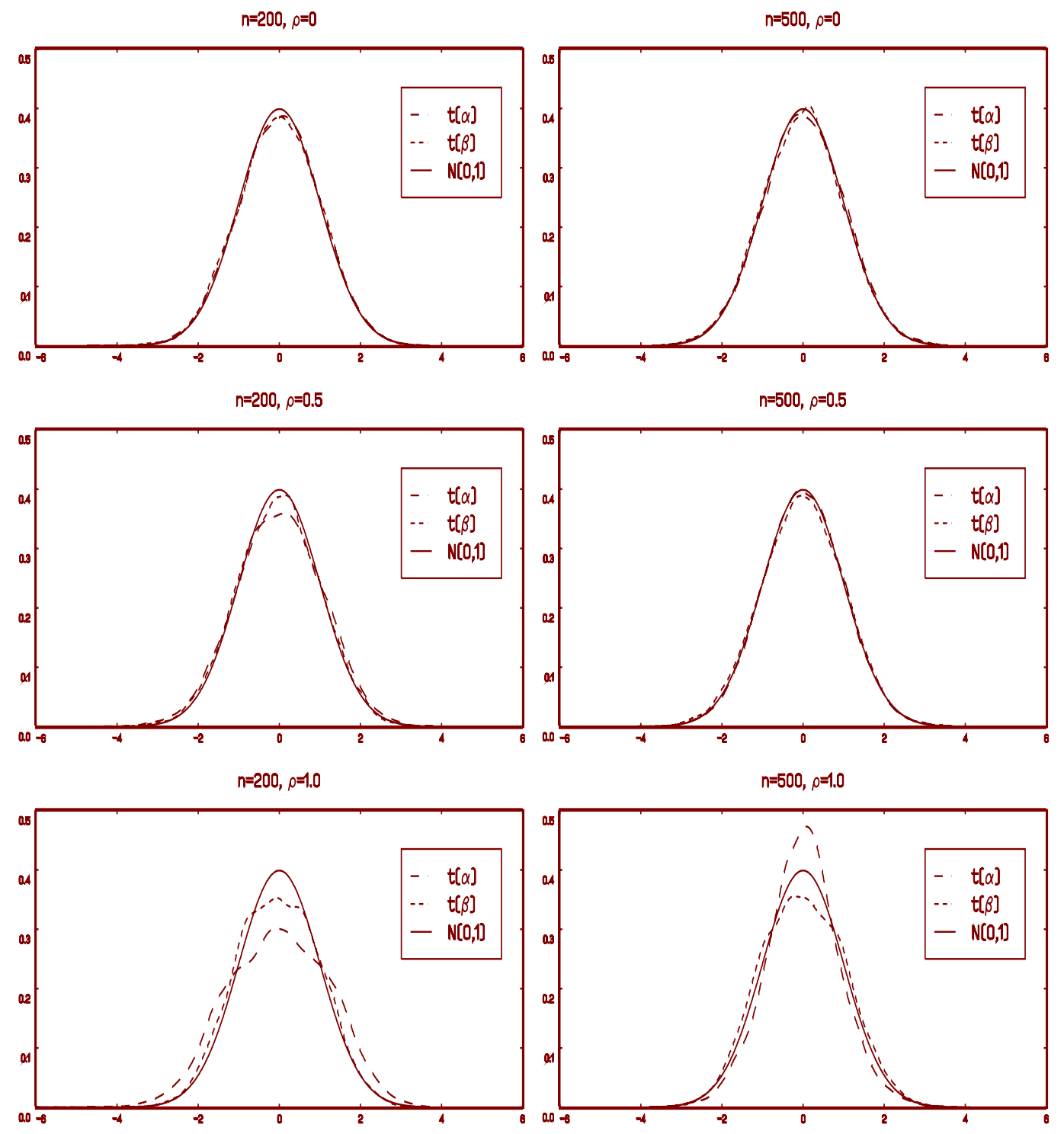

Figure 6: Densities of NLS $t$-ratios, Power Function, $\beta_{0}=2$ 

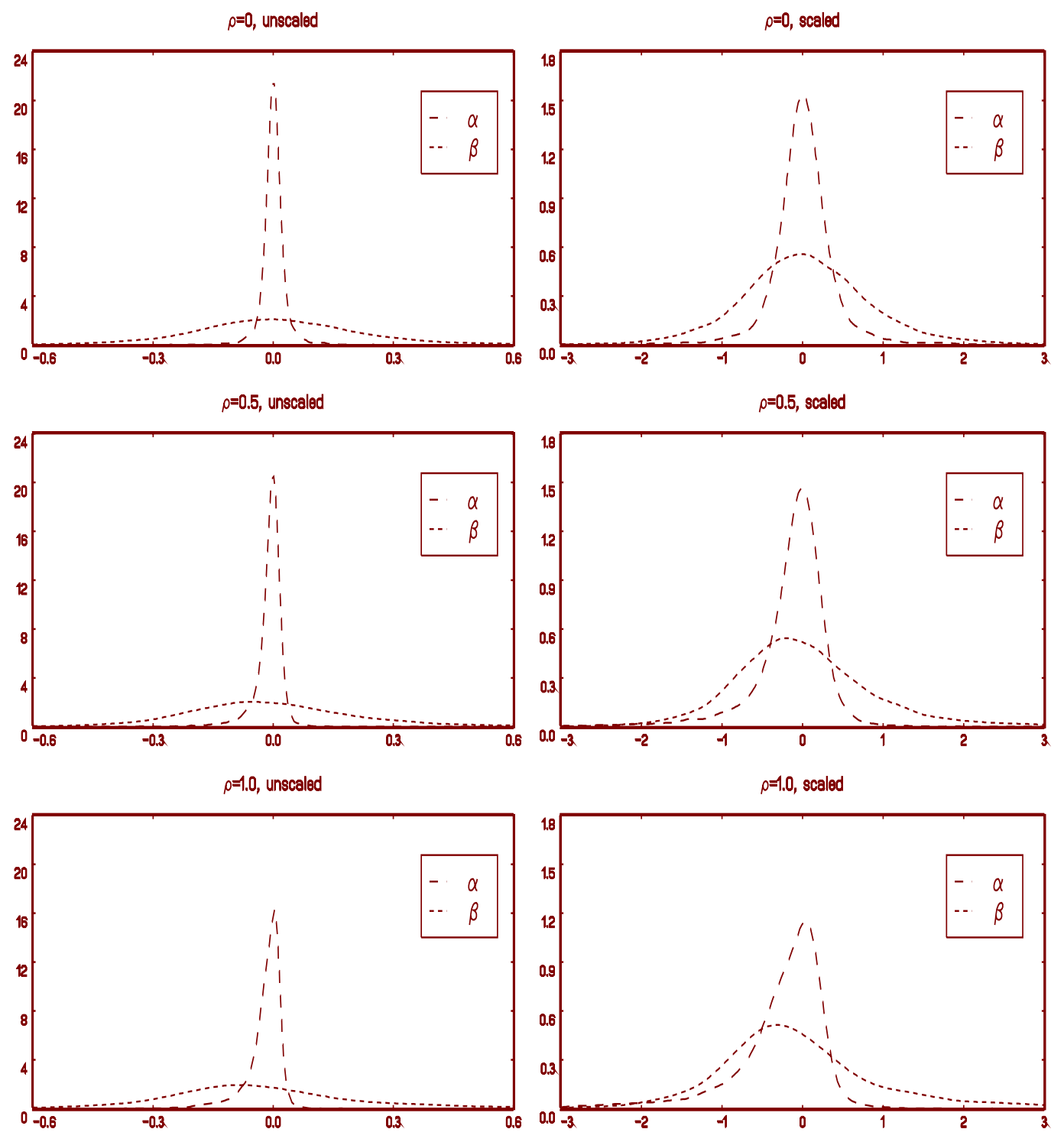

Figure 7: Densities of NLS Estimators, Logistic Function, $n=200$ 

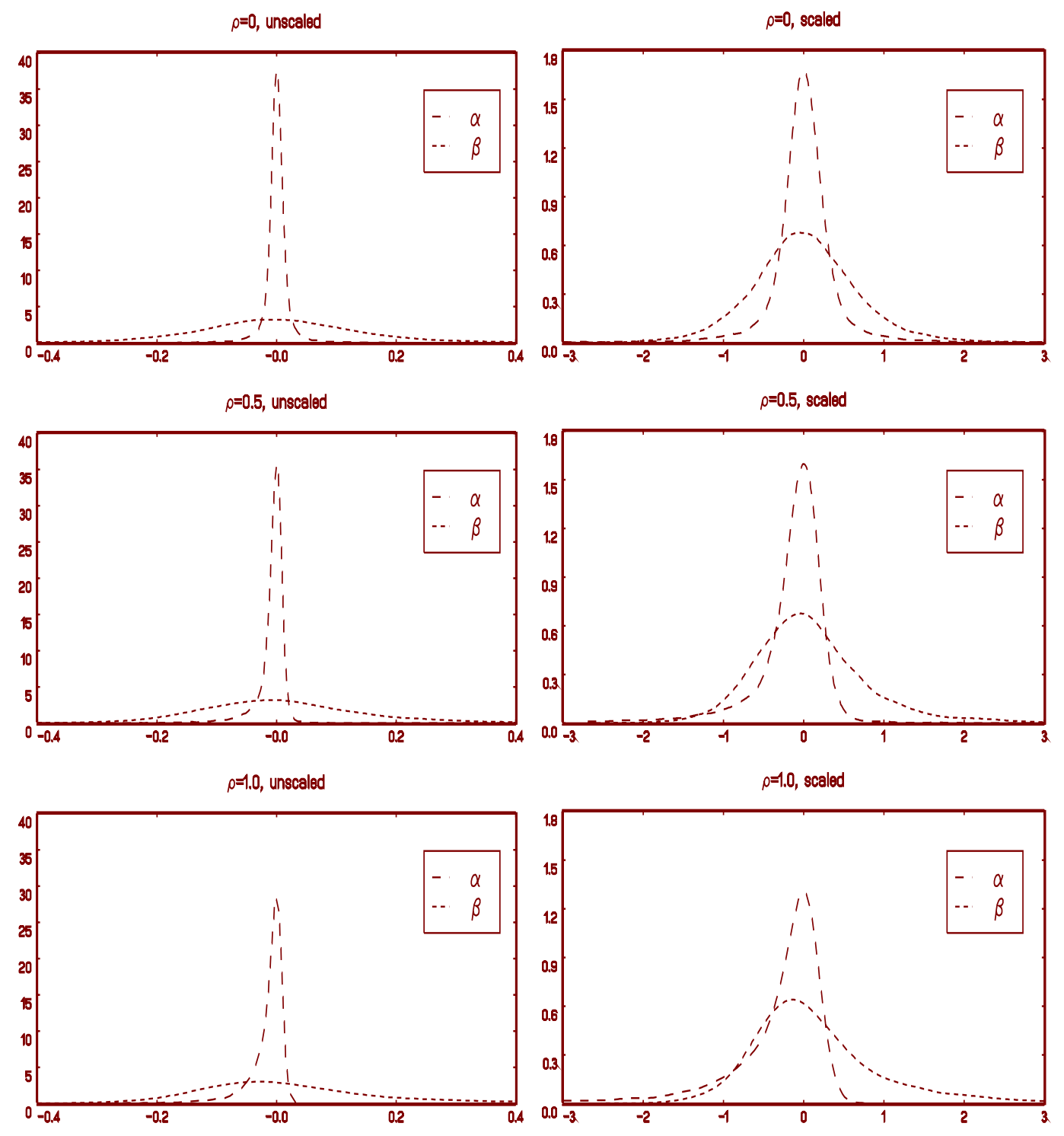

Figure 8: Densities of NLS Estimators, Logistic Function, $n=500$ 

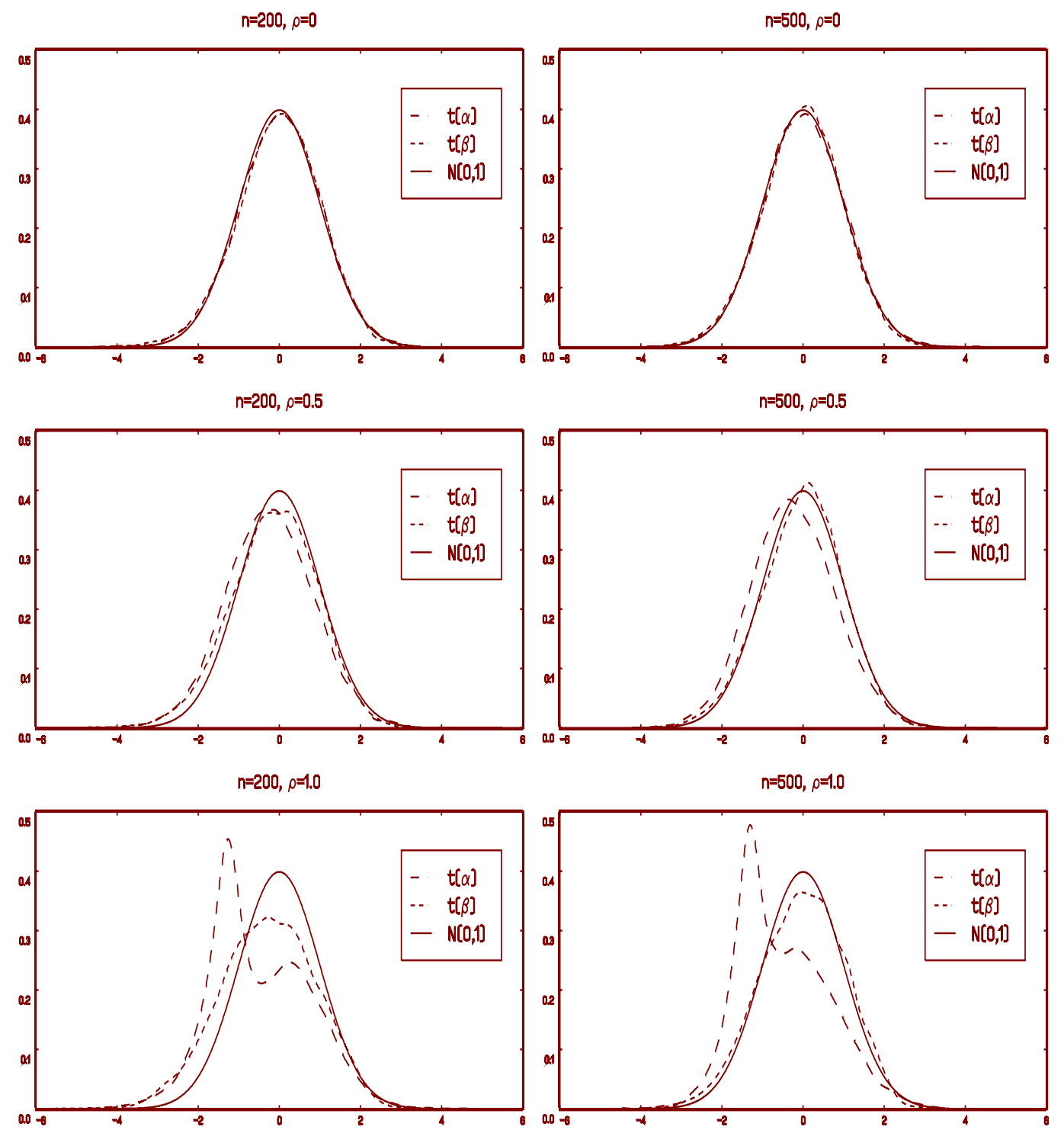

Figure 9: Densities of NLS $t$-ratios, Logistic Function 